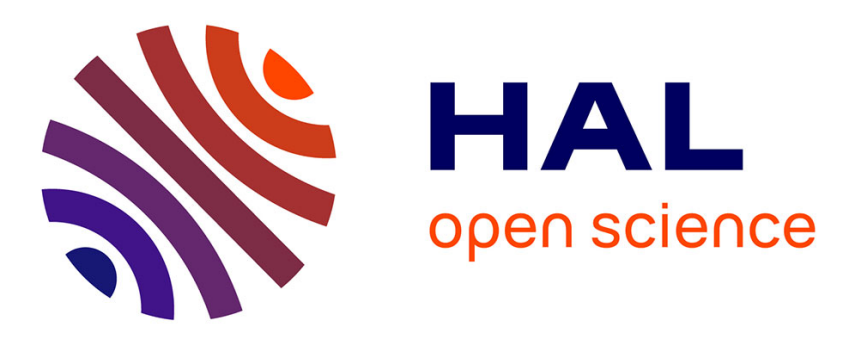

\title{
An Improved Characteristic Based Volume Penalization Method for the Euler Equations Towards Icing Applications
}

Pierre Lavoie, Emmanuel Radenac, Ghislain Blanchard, Éric Laurendeau, Philippe Villedieu

\section{To cite this version:}

Pierre Lavoie, Emmanuel Radenac, Ghislain Blanchard, Éric Laurendeau, Philippe Villedieu. An Improved Characteristic Based Volume Penalization Method for the Euler Equations Towards Icing Applications. Computers \& Fluids, 2021, 222, pp.104917. 10.1016/j.compfluid.2021.104917 . hal$02872954 \mathrm{v} 2$

\section{HAL Id: hal-02872954 \\ https://hal.science/hal-02872954v2}

Submitted on 7 Apr 2021

HAL is a multi-disciplinary open access archive for the deposit and dissemination of scientific research documents, whether they are published or not. The documents may come from teaching and research institutions in France or abroad, or from public or private research centers.
L'archive ouverte pluridisciplinaire HAL, est destinée au dépôt et à la diffusion de documents scientifiques de niveau recherche, publiés ou non, émanant des établissements d'enseignement et de recherche français ou étrangers, des laboratoires publics ou privés. 


\title{
An Improved Characteristic Based Volume Penalization Method for the Euler Equations Towards Icing Applications
}

\author{
Pierre Lavoie $^{\mathrm{a}, \mathrm{b}, *}$, Emmanuel Radenac ${ }^{\mathrm{b}}$, Ghislain Blanchard ${ }^{\mathrm{b}}$, Éric \\ Laurendeau ${ }^{\mathrm{a}}$, Philippe Villedieu ${ }^{\mathrm{b}}$ \\ ${ }^{a}$ Department of Mechanical Engineering, Polytechnique Montréal, C.P. 6079, succ. \\ Centre-ville, Montréal (Qc), H3C 3A7, Canada \\ ${ }^{b}$ ONERA - The French Aerospace Lab, F-31055 Toulouse, France
}

\begin{abstract}
Immersed boundary methods (IBMs) are an interesting alternative to the usual body-fitted mesh approach when dealing with complex geometries as they allow simpler mesh generation. The volume penalization method (an IBM) is commonly used for incompressible and compressible viscous flows but only one application to compressible inviscid flows can be found, which uses the characteristicbased volume penalization (CBVP) method. This approach penalizes the Euler equations to enforce a no-penetration velocity and an adiabatic wall while accounting for wall curvature. A new penalization method based on the CBVP is proposed to impose the conservation of entropy and total enthalpy in the normal direction to the wall instead of the classical adiabatic condition. The two approaches are compared and numerically tested on several cases: weakly compressible flow around a circular cylinder, subsonic flow around a NACA0012 airfoil and flow around a challenging high curvature ice horn. The new method is found to be more accurate than the CBVP on coarser meshes and better at retrieving attached flows for curved geometries. The paper concludes that the proposed method is suitable for general aerospace applications and beneficial for icing simulations which can exhibit highly curved geometries.
\end{abstract}

\footnotetext{
* Corresponding author

Email address: pierre.lavoie@polymtl.ca (Pierre Lavoie)
} 
Keywords: CFD, Euler Equations, Volume Penalization, Compressible Flow, Inviscid Flow, Immersed Boundary Method, Icing, Ice Accretion

\section{Introduction}

Immersed boundary methods (IBM) are an interesting alternative to the usual body-fitted (BF) mesh approach when dealing with complex and moving geometries. With BF meshes, the boundary conditions can be imposed exactly but the mesh generation is more restrictive as it must conform to the geometry. Alternatively when using an IBM, the geometry can arbitrarily cut through the mesh, even allowing the use of Cartesian grids which are simpler to generate and allow the use of fast and efficient algorithms [1. On the other hand, effort must be spent on the correct imposition of the boundary conditions.

Amongst the variety of IBMs available in the literature (see for instance 1 or [2] for a review), continuous IBMs have the advantage of being independent of the discretization and numerical methods. Furthermore, continuous approaches such as the volume penalization method of [3] are appealing for their simplicity of implementation. This type of approach stems from the Brinkman penalization method [4] where a penalization term is applied as a basic source term in the momentum equation of the incompressible Navier-Stokes equations to account for the presence of a porous medium. The idea was generalized for solid bodies in [3, 5], where the no-slip wall boundary condition is applied by assuming a porous medium with very low permeability. An analysis of the method is also given in [3] along with error estimates for the penalization. The Brinkman penalization or volume penalization was applied for instance in [6] for the tracking of ice shedding trajectories in a Cartesian grid, again using an incompressible laminar viscous flow.

For compressible viscous flow, a penalization term is added to the continuity equation in [7. The same approach is employed by [8] where a comparison between the penalization and direct-forcing method (another type of IBM) is performed. In [9], the momentum equation is penalized along with the energy 
equation in order to apply a fixed wall temperature, but the continuity equation is left untouched. A similar approach is followed by [10, 11].

A generalization of the Brinkman penalization method is proposed by [12] where the imposition of Dirichlet, Neumann and Robin boundary conditions is discussed for diffusion and convection-diffusion problems. Another generalization is proposed by [13, the characteristic-based volume penalization (CBVP) method, which provides a systematic way of implementing Dirichlet, Neumann and Robin conditions using source terms and hyperbolic penalization terms.

As shown by the previous literature review, the Brinkman penalization method is commonly used with incompressible or compressible viscous flows and rare applications to compressible inviscid flows can be found. The Brinkman penalization is applied to the prediction of acoustic scattering in [14] using the linearized Euler equations. The slip wall boundary condition is achieved by penalization of the normal velocity component (on the momentum equation). For aerodynamics, only one application is found in the literature where the Euler equations are penalized [15] using the method of [13].

Contrary to the penalization of the Navier-Stokes equations, the penalization of the Euler equations involves only one component of the velocity $(\mathbf{v} \cdot \mathbf{n})$ for the wall boundary condition. Without proper treatment, the continuity of the other variables at the wall is not ensured and can pollute the near-wall solution and thus the wall data extraction. This problem is not observed for Navier-Stokes equation which exhibits its own set of issues related to the characteristics of the boundary layer ( e.g. flow anisotropy, strong gradients).

In this paper, the Characteristic-Based Volume Penalization (CBVP) of 13, 15 is applied to the Euler equations in an alternative way. Our approach uses a different set of boundary conditions inspired by [16] where the conservation of entropy and total enthalpy in the normal direction are applied. This set of boundary conditions also accounts for the wall curvature and allows superior mesh convergence for curved geometries [16]. This type of boundary condition is useful for general applications and was found beneficial for the numerical simulation of ice accretion where ice shapes can exhibit features of high curvature. 
It is worth mentioning that the Euler equations are still relevant in the icing community where many numerical tools for industrial applications are based on inviscid-viscous coupling (e.g. LEWICE [17], IGLOO2D [18]). The Euler equations can also be used during the preliminary design of aerodynamic shapes (with or without viscous-inviscid interaction). For instance, [19] developed a ghost-cell method (a type of IBM) on Cartesian grids following this goal.

For this paper, the implementation of the penalization method is performed in the Euler flow solver of ONERA's 2D icing suite: IGLOO2D [18]. Although higher benefits are achieved when using IBMs on Cartesian grids in terms of computational effort and mesh generation, unstructured meshes are used in this paper for more flexibility in mixing body-fitted and immersed boundary approaches. It allows the simultaneous use of the two approaches within a simulation and also help in comparing the IBM to the body-fitted approach as the numerical code is against itself. Moreover, the penalization method is independent of the discretization and therefore the developments made in this paper are equally applicable to Cartesian, structured and unstructured meshes. The penalization methods are presented in $2 \mathrm{D}$ but their extension to $3 \mathrm{D}$ does not present any new difficulty as the evaluation of the curvature in 3D (a key element of the method) has already been treated in the literature [20, 15.

The paper starts with the review of two types of numerical wall boundary conditions for the Euler equations and their application to body-fitted meshes in a finite volume context. This is helpful to understand the boundary conditions that are to be imposed with the penalization method. Then in section 3 , the representation of the immersed boundary is discussed. Section 4 is dedicated to the description of the penalization method. More precisely, the penalization method of [13] and its application to the Euler equations [15] is discussed. Then the development of the new penalization method is presented along with implementation details for a Finite Volume Method. In section 5, verification of the new method is made on canonical test cases and on a challenging 2D ice horn case. Some comparisons are also made against the CBVP method. 


\section{Wall Boundary Conditions for the Euler Equations}

In this section, wall boundary conditions for the Euler equations are reviewed for motionless body-fitted meshes. The Euler equations are reminded in both conservative and non-conservative forms as both formulations are used later in this paper. Then two types of numerical wall boundary conditions are described for finite volume implementation.

The non-conservative form of the Euler equations is:

$$
\begin{array}{r}
\frac{\partial \rho}{\partial t}+\rho \boldsymbol{\nabla} \cdot \mathbf{v}+\mathbf{v} \cdot \nabla \rho=0 \\
\rho \frac{\partial \mathbf{v}}{\partial t}+\rho \mathbf{v} \cdot \nabla \mathbf{v}+\nabla P=0 \\
\rho \frac{\partial e}{\partial t}+\rho \mathbf{v} \cdot \nabla e+P \boldsymbol{\nabla} \cdot \mathbf{v}=0
\end{array}
$$

and its conservative form is written as:

$$
\begin{aligned}
\frac{\partial \rho}{\partial t}+\nabla \cdot(\rho \mathbf{v}) & =0 \\
\frac{\partial(\rho \mathbf{v})}{\partial t}+\nabla \cdot(\rho \mathbf{v} \otimes \mathbf{v}+P \mathbf{I}) & =0 \\
\frac{\partial(\rho E)}{\partial t}+\nabla \cdot((\rho E+P) \mathbf{v}) & =0
\end{aligned}
$$

where $\rho$ is the density, $\mathbf{v}$ the velocity, $P$ the pressure, $e$ the internal energy, $E$ the total energy, $H$ is the total enthalpy and $\mathbf{I}$ the identity tensor.

$$
\begin{aligned}
e & =\frac{R}{\gamma-1} T \\
E & =e+\frac{1}{2}\|\mathbf{v}\|^{2} \\
H & =\gamma e+\frac{1}{2}\|\mathbf{v}\|^{2}
\end{aligned}
$$

The system is closed using the ideal gas law.

$$
P=\rho R T
$$

The specific gas constant for air is $R=287.0$ and the specific heat ratio is $\gamma=1.4$.

The wall boundary conditions for the Euler equations are set in order to obtain a no-penetration velocity (or slip velocity) where $\mathbf{v} \cdot \mathbf{n}=0$ (the boundaries 
are assumed motionless for this paper). This leads to the following wall flux (in 2D):

$$
\mathbf{F}_{w a l l}=\left[\begin{array}{c}
\rho \mathbf{v} \cdot \mathbf{n} \\
\rho u \mathbf{v} \cdot \mathbf{n}+P n_{x} \\
\rho v \mathbf{v} \cdot \mathbf{n}+P n_{y} \\
\rho H \mathbf{v} \cdot \mathbf{n}
\end{array}\right]=\left[\begin{array}{c}
0 \\
P n_{x} \\
P n_{y} \\
0
\end{array}\right]
$$

On a physics point of view, only the no-penetration velocity is a required boundary condition at the wall. However on a numerical point of view (i.e. finite volume method), adequate values for the variables $\rho, u, v, P$ are required in the ghost cells for both the evaluation of the wall flux and the evaluation of the gradients (MUSCL reconstruction). Two approaches are reviewed here, which are both implemented in the unstructured Euler flow solver of IGLOO2D: the Symmetry Technique (ST) and the Curvature Corrected Symmetry Technique (CCST).

\subsection{Symmetry Technique (ST)}

Considering a Finite Volume cell-centered discretization using ghost cells at the wall boundary (Figure 1), the Symmetry Technique consists in imposing the following variables in the ghost cells $(\mathrm{g})$ to obtain the appropriate wall flux:

$$
\begin{aligned}
& \mathbf{v}_{g}=\mathbf{v}_{d}-2\left(\mathbf{v}_{d} \cdot \mathbf{n}\right) \mathbf{n} \\
& \rho_{g}=\rho_{d} \\
& P_{g}=P_{d}
\end{aligned}
$$

where $\mathbf{n}$ is the wall normal. It follows the assumption that the wall is locally flat (negligible curvature). This assumption holds if the mesh in the vicinity of the wall is sufficiently refined (see Eq. 12 with $\Delta n=0$ ). 


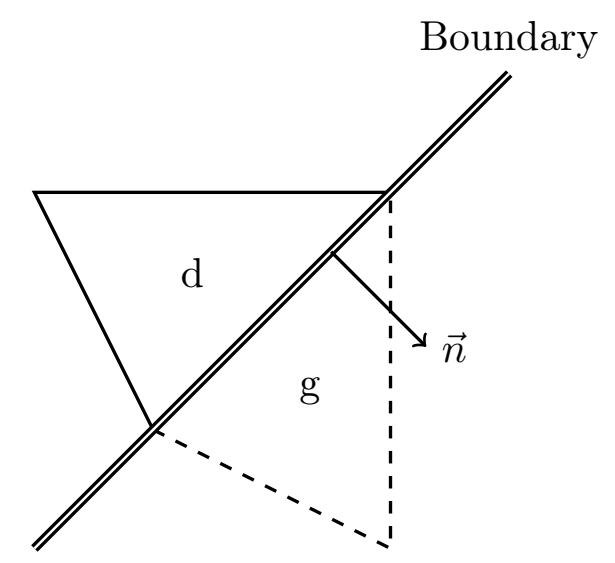

Figure 1: Representation of the domain (d) and ghost (g) cells at the boundary along with the wall normal $(\mathbf{n})$

\subsection{Curvature Corrected Symmetry Technique (CCST)}

The CCST is presented in 2D in [16, 21], then extended to 3D in [20] and applied to 2D unstructured meshes in [22]. This approach imposes the normal momentum relation to account for the wall curvature in the pressure extrapolation, which can be written as:

$$
\left.\frac{\partial P}{\partial \mathbf{n}}\right|_{w}=\rho_{w} \kappa\left\|\mathbf{v}_{w}\right\|^{2}
$$

where $\kappa$ is the signed wall curvature (positive if the center of curvature is on the domain/fluid side, negative on the ghost/solid side). Note that Eq. (11) is derived from the momentum equation and is valid for both steady and unsteady flows. However its application is limited to stationary and non-deformable bodies $\left(\frac{\partial \mathbf{n}}{\partial t}=0\right)$. As a consequence of Eq. 11 the ghost cell values are computed as:

$$
\begin{aligned}
P_{g} & =P_{d}+\rho_{w} \kappa\left\|\mathbf{v}_{w}\right\|^{2} \Delta n \\
\rho_{g} & =\rho_{d}\left(\frac{P_{g}}{P_{d}}\right)^{1 / \gamma} \\
(\mathbf{v} \cdot \mathbf{n})_{g} & =-(\mathbf{v} \cdot \mathbf{n})_{d} \\
(\mathbf{v} \cdot \mathbf{t})_{g}^{2} & =(\mathbf{v} \cdot \mathbf{t})_{d}^{2}+\frac{2 \gamma}{\gamma-1}\left(\frac{P_{d}}{\rho_{d}}-\frac{P_{g}}{\rho_{g}}\right)
\end{aligned}
$$


where $\Delta n$ is the distance between the centers of the domain (d) and ghost (g) cells along the normal direction. Eq. (13) and Eq. (15) result from the conservation of entropy and total enthalpy respectively. The wall $(w)$ values in Eq. 12 are typically taken as [16, 22]:

$$
\begin{aligned}
\rho_{w} & =\rho_{d} \\
\mathbf{v}_{w} & =\mathbf{v}_{d}-\left(\mathbf{v}_{d} \cdot \mathbf{n}\right) \mathbf{n}
\end{aligned}
$$

The norm of the tangential velocity is computed from Eq. (15) and its direction follows the unit tangent vector computed as:

$$
\mathbf{t}=\frac{\mathbf{v}-(\mathbf{v} \cdot \mathbf{n}) \mathbf{n}}{\|\mathbf{v}-(\mathbf{v} \cdot \mathbf{n}) \mathbf{n}\|}
$$

The CCST is shown to generate less numerical entropy and to exhibit faster grid convergence for steady flow computations around a cylinder [16]. However, the ST and CCST boundary conditions converge towards the same solution as the mesh is refined. Furthermore, when the curvature is zero $(\kappa=0)$, the CCST method simplifies to the ST approach.

To assess if a simulation benefited from the CCST, the correction term $\left(\rho_{w} \kappa\left\|\mathbf{v}_{w}\right\|^{2} \Delta n\right)$ in the pressure extrapolation (Eq. 12 $)$ can be evaluated. It can be interpreted as the error $(\Delta P$, Eq. $19 p)$ committed on the pressure extrapolation when using the ST instead of the CCST, where the characteristic wall cell size $(\Delta x)$ is used to estimate $\Delta n$. Using the relative error Eq. (20), one can observe that it depends not only on curvature and cell size, but also on the local wall Mach number $\left(M_{w}\right)$. Thus a scenario with high curvature and a coarse mesh would greatly benefit from the use of the CCST.

$$
\begin{aligned}
& \Delta P=\rho_{w}\left\|\mathbf{v}_{w}\right\|^{2} \kappa \Delta x \\
& \frac{\Delta P}{P_{w}}=\gamma M_{w}^{2} \kappa \Delta x
\end{aligned}
$$

\section{Immersed Boundary Representation}

Before describing the penalization methods for the Euler equations, it is worth discussing how the immersed boundary (IB) is represented. 


\subsection{Signed Distance}

For this paper, the immersed boundary (IB) is defined by a discrete list of nodes $(2 \mathrm{D})$. The location of the IB is defined by the level-set $\phi=0$, where $\phi$ is the signed distance field from the IB. Values of $\phi$ are computed using a geometric approach : evaluating the minimum projected distance to the edges forming the IB $[23$. The signed distance is negative $(\phi<0)$ in the solid and positive in the fluid $(\phi>0)$.

Using a signed distance field leads to a simple evaluation of the normals $\left(\mathbf{n}_{\phi}\right)$ and curvature $(\kappa)$ of the IB using:

$$
\begin{gathered}
\mathbf{n}_{\phi}=-\frac{\boldsymbol{\nabla} \phi}{\|\boldsymbol{\nabla} \phi\|} \\
\kappa=\boldsymbol{\nabla} \cdot \mathbf{n}_{\phi} .
\end{gathered}
$$

Note that the normal based on $\phi$ has a negative sign in order to point towards the solid zone $(\phi<0)$. This is useful in the definition of the penalization methods presented in the next section.

\subsection{Data Extraction}

To extract the data at the IB (e.g. density, velocity, pressure), a weighted least square interpolation at the discrete nodes defining the IB is used. The weight is based on the inverse distance with a threshold on the distance $(0.5 \Delta x)$ to avoid division by a very small number and provide some smoothing to the data. The interpolation stencil is determined firstly by identifying the cell containing the IB node, and secondly by saving the extended neighborhood of this cell. The penalization methods described in the following sections extend the fluid data into the solid zone (from outside the geometry to its inside). Thus the interpolation stencil in the vicinity of the solid/fluid interface is assumed to be filled with valid data to perform the interpolation.

\section{Penalization Method}

In this section, the volume penalization method [3, 5] is presented along with a discussion on its application to the Euler equations. Then the penalization 
method of [13] (CBVP) is described followed by the development of the improved penalization approach called CBVP-Hs because it conserves total enthalpy $(\mathrm{H})$ and entropy (s).

\subsection{Volume Penalization}

The volume penalization method consists in adding source terms in the continuous form of the equation to enforce the desired boundary condition. The source terms are activated/deactivated using a mask function $(\chi)$ equal to unity in the solid and zero in the fluid. In this way, only the solid is penalized and the usual equations are retrieved in the fluid. The penalization parameter $(\eta \ll 1)$ ensures the boundary condition is enforced accurately. This type of method is mostly used for Dirichlet boundary conditions but a general extension of the method to Neumann and Robin conditions is proposed in [12]. The volume penalization is limited to $1^{\text {st }}$ order accuracy in space [24]. However, a $2^{\text {nd }}$ order adaptation of the method, the sub-mesh penalty method, is proposed by [24].

The volume penalization is widely used for the Navier-Stokes equations where the velocity $\mathbf{v}$ is penalized on the momentum equations to obtain $\mathbf{v}=0$ in the solid (for a stationary body). Some authors also apply penalization terms to the density and energy equation for compressible flows (e.g. [7],9]). Other implementations for adiabatic walls do not require the penalization of these equations (e.g. [10]). A simple adaptation of this approach to the Euler equations consists in penalizing only the momentum equations to obtain $\mathbf{v} \cdot \mathbf{n}=0$ in the solid instead, similar to [14]. In brief, the goal is to obtain a slip velocity in the solid instead of the no-slip condition. In non-conservative form the penalized Euler equations read:

$$
\begin{gathered}
\frac{\partial \rho}{\partial t}+\boldsymbol{\nabla} \cdot(\rho \mathbf{v})=0 \\
\rho \frac{\partial \mathbf{v}}{\partial t}+\rho \mathbf{v} \cdot \nabla \mathbf{v}+\nabla P=-\frac{\chi}{\eta} \rho\left(\mathbf{v} \cdot \mathbf{n}_{\phi}\right) \mathbf{n}_{\phi} \\
\rho \frac{\partial e}{\partial t}+\rho \mathbf{v} \cdot \nabla e+P \boldsymbol{\nabla} \cdot \mathbf{v}=0
\end{gathered}
$$

In the fluid $(\chi=0)$, the usual Euler equations are retrieved. In the solid the penalization term is activated by $\chi=1$. As $\eta \ll 1$, the physical terms in the 
momentum equation are negligible in front of the penalization term. It thus comes back to solving an ordinary differential equation of the form:

$$
\frac{d \mathbf{v}}{d t}=-\frac{1}{\eta}\left(\mathbf{v} \cdot \mathbf{n}_{\phi}\right) \mathbf{n}_{\phi}
$$

where the penalization parameter $\eta$ can be seen as a characteristic timescale. The solution of this ODE is a rapidly decaying exponential (see Eq. 25 for the $\mathrm{x}$ component), which means the no-penetration velocity is imposed almost instantaneously (Eq. 26)).

$$
\begin{aligned}
\left(\mathbf{v} \cdot \mathbf{n}_{\phi}\right) n_{\phi, x} & =A_{0} e^{-\left(t n_{\phi, x}\right) / \eta} \\
\left(\mathbf{v} \cdot \mathbf{n}_{\phi}\right) \mathbf{n}_{\phi} & =0
\end{aligned}
$$

The Euler equations are also solved in the solid but because of Eq. 26, , only the tangential component of the velocity remains $\left(\mathbf{v}_{t}\right)$ :

$$
\begin{array}{r}
\frac{\partial \rho}{\partial t}+\nabla \cdot\left(\rho \mathbf{v}_{t}\right)=0 \\
\rho \frac{\partial \mathbf{v}_{t}}{\partial t}+\rho \mathbf{v}_{t} \cdot \nabla \mathbf{v}_{t}+\nabla P=0 \\
\rho \frac{\partial e}{\partial t}+\rho \mathbf{v}_{t} \cdot \nabla e+P \nabla \cdot \mathbf{v}_{t}=0
\end{array}
$$

In [15], more complex penalized Euler equations are briefly suggested but no justification for their selection is provided. Numerical experiments showed that with a simpler form like Eq. (23), the velocity tends to zero inside the solid and an artificial boundary layer is created near the immersed boundary. This behavior was unexpected as, contrary to the Navier-Stokes equations where the fluid is at rest in the solid (no-slip wall), Eq. (27) shows that there should be a tangential fluid flow in the solid with the Euler equations (slip wall). Because the no-penetration velocity is imposed at the IB and because there is no diffusion term, there is a lack of communication between the fluid and the solid. One constraint only is imposed: the IB is a streamline because it is parallel to the flow. But some discontinuities in tangential velocity $\left(\mathbf{v}_{t}\right)$, pressure, entropy and total enthalpy are allowed across the IB, which is thus a slip line. The objective is then to retrieve continuity across the streamline at the IB by the imposition 
of additional constraints in the normal direction. This can be achieved by enforcing Neumann boundary conditions, which can be implemented using the penalization method of [13], presented in the following section.

\subsection{Characteristic-Based Volume Penalization (CBVP)}

The Characteristic-based Volume Penalization (CBVP) method [13] provides a systematic way of implementing Dirichlet, Neumann and Robin boundary conditions by the addition of hyperbolic penalization terms. It uses a sharp Heaviside function for the mask $(\chi)$ where $\chi=1$ in the solid $\left(\Omega_{s}\right)$ and $\chi=0$ in the fluid $\left(\Omega_{f}\right)$. This leads to a staircase definition of the immersed boundary (IB) as shown in Figure 2. However, a smooth solution is recovered at the IB by the use of hyperbolic penalization terms for Neumann and Robin conditions, and by the use of dissipation terms for Dirichlet conditions.

\begin{tabular}{|l|l|l|}
\hline & & \multicolumn{1}{|c|}{} \\
\hline & & \\
\hline & & \\
\hline
\end{tabular}

Figure 2: Staircase representation of the solid

In [15, a set of penalized Euler equations are presented very briefly. In non-conservative form, it writes:

$$
\begin{aligned}
& \frac{\partial \rho}{\partial t}+(1-\chi) \boldsymbol{\nabla} \cdot(\rho \mathbf{v})=-\frac{\chi}{\eta_{c}}\left(\mathbf{n}_{\phi} \cdot \boldsymbol{\nabla} \rho-\kappa \frac{\rho^{2}}{P}\|\mathbf{v}\|^{2}\right) \\
& \rho \frac{\partial \mathbf{v}}{\partial t}+(1-\chi)(\rho \mathbf{v} \cdot \boldsymbol{\nabla} \mathbf{v}+\boldsymbol{\nabla} P)=-\frac{\chi}{\eta} \rho\left(\mathbf{v} \cdot \mathbf{n}_{\phi}\right) \mathbf{n}_{\phi}+\chi \rho \nu_{\eta} \boldsymbol{\nabla}^{2} \mathbf{v} \\
& \rho \frac{\partial e}{\partial t}+(1-\chi)(\rho \mathbf{v} \cdot \boldsymbol{\nabla} e+P \boldsymbol{\nabla} \cdot \mathbf{v})=-\frac{\chi}{\eta_{c}}\left(\rho \mathbf{n}_{\phi} \cdot \boldsymbol{\nabla} e\right)
\end{aligned}
$$

where $\mathbf{n}_{\phi}$ is the normal to the IB pointing towards the solid. Note that $1 / \eta_{c}$ can be seen as a characteristic velocity with $\eta_{c} \ll 1$. Here, hyperbolic penalization 
terms of the form $(\mathbf{n} \cdot \nabla u-q) / \eta_{c}=0$ are added to the continuity and energy equations to apply Neumann boundary conditions. As explained in [13], for the CBVP method the physical flux terms are removed from $\Omega_{s}$ to prevent any interaction with the penalization terms. For Dirichlet conditions, an artificial dissipation term is added using the artificial viscosity $\nu_{\eta}$ which must be of the order $\nu_{\eta} \geq \Delta x^{2} / \eta$. According to [13], this term helps in retrieving a smooth solution at the IB for Dirichlet conditions.

In the fluid $(\chi=0)$, the usual Euler equations are retrieved. In the solid $(\chi=1)$, by setting the time derivative to zero (steady state), the following conditions are enforced:

$$
\begin{aligned}
\mathbf{n}_{\phi} \cdot \boldsymbol{\nabla} \rho & =\kappa \frac{\rho^{2}}{P}\|\mathbf{v}\|^{2} \\
-\frac{1}{\eta} \rho\left(\mathbf{v} \cdot \mathbf{n}_{\phi}\right) \mathbf{n}_{\phi}+\nu_{\eta} \nabla^{2} \mathbf{v} & =0 \\
\mathbf{n}_{\phi} \cdot \nabla e & =0
\end{aligned}
$$

This set of equations is equivalent to imposing an adiabatic wall (Eq. (31)), a no penetration velocity (Eq. (30) ) and the normal momentum relation which relates the pressure gradient to the wall curvature (Eq. (29)), except it is written in terms of the density gradient. The normal momentum relation can be written as:

$$
\mathbf{n}_{\phi} \cdot \nabla P=\kappa \rho\|\mathbf{v}\|^{2}
$$

where in $2 \mathrm{D}$, the curvature $(\kappa)$ is computed from the normals to the IB $\left(\mathbf{n}_{\phi}\right)$ as $\kappa=\boldsymbol{\nabla} \cdot \mathbf{n}_{\phi}$. To obtain Eq. (29), one must use the ideal gas law $(P=\rho e(\gamma-1))$ in combination with the normal momentum relation (Eq. (32)) and apply the adiabatic wall condition $\left(\mathbf{n}_{\phi} \cdot \nabla e=0\right)$.

The dissipation term in Eq. (30) helps in obtaining continuity of the tangential velocity but also hinders the imposition of $(\mathbf{v} \cdot \mathbf{n}) \mathbf{n}=0$. Thus this parameter must be selected small enough for the no-penetration velocity to be enforced, but large enough to ensure continuity and stability. In this paper the dissipation parameter is taken as $\nu_{\eta}=\Delta x^{2} / \eta$ as suggested in [13]. Note that this penalization method depends on three adjustable parameters: $\eta, \eta_{c}$ and $\nu_{\eta}$ 
instead of one for the volume penalization method.

\section{3. $\mathrm{CBVP}-\mathrm{Hs}$}

Because of the dissipation term in the CBVP method, there is a smooth transition of the velocity from the fluid to the solid. However, this transition is not based on the physics of the problem and does not ensure the conservation of total enthalpy. A new penalization of the Euler equations is proposed hereafter, where the CBVP method of [13] is used to apply the wall boundary conditions of the CCST [16, 21, 20].

The goal is to develop a method that would respect the properties of the inviscid ideal gas flow in the vicinity of the wall streamline. The streamline is, of course, defined by the no-penetration velocity (Eq. (36)). The continuity of tangential velocity, pressure, entropy and total enthalpy must be ensured by additional equations. The normal momentum relation (Eq. (32) allows the continuity of pressure. The normal conservation of total enthalpy (Eq. (33)) and the normal conservation of entropy (Eq. (34) are imposed to close the system:

$$
\begin{aligned}
\mathbf{n}_{\phi} \cdot \nabla H & =0 \\
\mathbf{n}_{\phi} \cdot \nabla s & =0 \\
\mathbf{n}_{\phi} \cdot \nabla P & =\kappa \rho\|\mathbf{v}\|^{2} \\
\left(\mathbf{v} \cdot \mathbf{n}_{\phi}\right) \mathbf{n}_{\phi} & =0
\end{aligned}
$$

where $\mathbf{n}_{\phi}$ is the normal to the IB based on the signed distance field $(\phi)$. This set of boundary conditions allows the continuity of the flow variables including the norm of the velocity across the boundary. It is better suited for homentropic and homenthalpic flows which are the primary target of our model, although the presence of a shock wave for instance may not hinder the use of (Eq. (34)) as long as the shock is parallel to $\mathbf{n}_{\phi}$.

Because this new method is based on the CBVP but designed to conserve the entropy (s) and total enthalpy $(\mathrm{H})$, it is referred to as CBVP-Hs. It is important to understand that the only physical wall boundary condition required for the Euler equations is the no-penetration velocity. The other conditions are 
numerical and are used to improve the accuracy of the model in terms of wall pressure extrapolation, generation of entropy and conservation of total enthalpy. The boundary conditions also improve the continuity of the solution near the IB and provide adequate support for the evaluation of the fluxes and gradients (e.g. for a 2nd order MUSCL approach). Furthermore, it allows for the use of an interpolation method for the extraction of the wall data.

In the following sections, penalization terms are first derived for the primitive variables and applied to the non-conservative form of the Euler equations. It is useful to start with the primitive variables as the penalization terms can easily be derived for them. Then the transition from the non-conservative to the conservative form naturally provides the penalized Euler equations in terms of conservative variables.

\subsubsection{Penalization of the Primitive Variables}

In this section, penalization terms are derived for the non-conservative form of the Euler equations (Eq. (1)). Hyperbolic penalization terms of the form $\mathbf{n} \cdot \boldsymbol{\nabla} u=q$ are sought, where $u$ is $\rho, \mathbf{v}$ or $e$.

The penalization term for the density is derived from Eqs. (34)-32. The conservation of entropy can be written as:

$$
\begin{aligned}
\mathbf{n}_{\phi} \cdot \nabla s & =\mathbf{n}_{\phi} \cdot \nabla\left(\frac{P}{\rho^{\gamma}}\right)=0 \\
\Rightarrow \mathbf{n}_{\phi} \cdot \nabla \rho & =\frac{\rho}{\gamma P} \mathbf{n}_{\phi} \cdot \nabla P
\end{aligned}
$$

By substituting the normal momentum relation (Eq. (32)) in Eq. (38), a relation for the normal density gradient is obtained. It is applied to the continuity equation as a hyperbolic penalization term (Eq. (39)).

$$
\frac{\chi}{\eta_{c}}\left(\mathbf{n}_{\phi} \cdot \nabla \rho-\frac{\rho^{2}}{\gamma P} \kappa\|\mathbf{v}\|^{2}\right)=0
$$

A penalization term for the internal energy can be derived from equations (6), (3) and (38), which gives:

$$
\frac{\chi}{\eta_{c}}\left(\mathbf{n}_{\phi} \cdot \nabla e-\frac{1}{\gamma} \kappa\|\mathbf{v}\|^{2}\right)=0
$$


At this point, only the penalization term on velocity is missing. This term is the application of the no-penetration velocity (Eq. (36)) as a Dirichlet condition and a hyperbolic penalization term denoted $\mathcal{P}_{\mathbf{v}}$ which must be determined to set the velocity magnitude.

$$
\begin{array}{r}
\rho \frac{\partial \mathbf{v}}{\partial t}+(1-\chi)(\rho \mathbf{v} \cdot \nabla \mathbf{v}+\nabla P)= \\
\mathcal{P}_{\mathbf{v}}-\frac{\chi}{\eta} \rho\left(\mathbf{v} \cdot \mathbf{n}_{\phi}\right) \mathbf{n}_{\phi}
\end{array}
$$

In addition, the conservation of total enthalpy must still be enforced. The term $\mathcal{P}_{\mathbf{v}}$ can thus be derived in two steps. First, by using the normal conservation of total enthalpy (Eq. (33p) one can derive a condition on kinetic energy. The conservation of total enthalpy $\left(\mathbf{n}_{\phi} \cdot \nabla H=0\right)$ can be written as:

$$
\frac{\gamma}{\gamma-1} \mathbf{n}_{\phi} \cdot \nabla\left(\frac{P}{\rho}\right)+\mathbf{n}_{\phi} \cdot \nabla\left(\frac{1}{2}\|\mathbf{v}\|^{2}\right)=0
$$

with

$$
\mathbf{n}_{\phi} \cdot \nabla\left(\frac{P}{\rho}\right)=\frac{\mathbf{n}_{\phi}}{\rho} \cdot \nabla P-\frac{P \mathbf{n}_{\phi}}{\rho^{2}} \cdot \nabla \rho
$$

By using Eq. (43), the normal momentum relation (Eq. (32)) and the condition on density (Eq. (39)), the condition on kinetic energy is retrieved.

$$
\Rightarrow \mathbf{n}_{\phi} \cdot \nabla\left(\frac{1}{2}\|\mathbf{v}\|^{2}\right)+\kappa\|\mathbf{v}\|^{2}=0
$$

It can also be recast in a penalized equation for the kinetic energy in the solid:

$$
\rho \frac{\partial\left(\frac{1}{2}\|\mathbf{v}\|^{2}\right)}{\partial t}=-\frac{\chi}{\eta_{c}}\left(\rho \mathbf{n}_{\phi} \cdot \nabla\left(\frac{1}{2}\|\mathbf{v}\|^{2}\right)+\rho \kappa\|\mathbf{v}\|^{2}\right)
$$

Second, a relation for kinetic energy can also be computed from the momentum equation (41) scalar product with $\mathbf{v}$ as:

$$
\begin{gathered}
\mathbf{v} \cdot \rho \frac{\partial \mathbf{v}}{\partial t}+\mathbf{v} \cdot(1-\chi)(\rho \mathbf{v} \cdot \nabla \mathbf{v}+\nabla P)= \\
\mathbf{v} \cdot \mathcal{P}_{\mathbf{v}}-\mathbf{v} \cdot \frac{\chi}{\eta} \rho\left(\mathbf{v} \cdot \mathbf{n}_{\phi}\right) \mathbf{n}_{\phi}
\end{gathered}
$$

By considering this equation in the solid $(\chi=1)$ and by keeping only terms of the same order of magnitude $\left(\eta \ll 1\right.$ and $\left.\eta \ll \eta_{c}\right)$, it reads:

$$
\mathbf{v} \cdot \rho \frac{\partial \mathbf{v}}{\partial t}=\mathbf{v} \cdot \mathcal{P}_{\mathbf{v}}
$$


or

$$
\rho \frac{\partial\left(\frac{1}{2}\|\mathbf{v}\|^{2}\right)}{\partial t}=\mathbf{v} \cdot \mathcal{P}_{\mathbf{v}}
$$

This simplification follows the assumption that $\eta$ and $\eta_{c}$ are not of the same order of magnitude and thus, that $\mathbf{v} \cdot \mathbf{n}_{\phi}$ exponentially tends towards zero. In order to equate Eq. (48) and Eq. 45, , $\mathcal{P}_{\mathbf{v}}$ must be selected to respect a condition on kinetic energy (Eq. 49p).

$$
\mathbf{v} \cdot \mathcal{P}_{\mathbf{v}}=-\frac{\chi}{\eta_{c}}\left(\rho \mathbf{n}_{\phi} \cdot \nabla\left(\frac{1}{2}\|\mathbf{v}\|^{2}\right)+\rho \kappa\|\mathbf{v}\|^{2}\right)
$$

A natural approach is to select $\mathcal{P}_{\mathbf{v}}$ orthogonal to $\mathbf{n}$ in order to decouple the hyperbolic penalization term and the Dirichlet condition (see Eq. 411). However, no practical formulation was found for use with the Finite Volume Method. An alternative and simpler choice for $\mathcal{P}_{\mathbf{v}}$ is:

$$
\mathcal{P}_{\mathbf{v}}=-\frac{\chi}{\eta_{c}}\left(\rho \mathbf{n}_{\phi} \cdot \nabla \mathbf{v}+\kappa \rho \mathbf{v}\right)
$$

However note that Eq. (50) is not the only possible choice for $\mathcal{P}_{\mathbf{v}}$. In Eq. 41), the hyperbolic penalization term (Eq. (50) and the Dirichlet condition (Eq. (36)) are decoupled by using $\eta \ll \eta_{c}$.

The non-conservative form of the penalized equations can now be updated using the hyperbolic terms for $\rho, \mathbf{v}$ and $e$ :

$$
\begin{aligned}
\frac{\partial \rho}{\partial t} & +(1-\chi) \boldsymbol{\nabla} \cdot(\rho \mathbf{v})=-\frac{\chi}{\eta_{c}}\left(\mathbf{n}_{\phi} \cdot \nabla \rho-\kappa \frac{\rho^{2}}{\gamma P}\|\mathbf{v}\|^{2}\right) \\
\rho \frac{\partial \mathbf{v}}{\partial t} & +(1-\chi)(\rho \mathbf{v} \cdot \nabla \mathbf{v}+\nabla P)=-\frac{\chi}{\eta_{c}}\left(\rho \mathbf{n}_{\phi} \cdot \nabla \mathbf{v}+\kappa \rho \mathbf{v}\right) \\
& -\frac{\chi}{\eta} \rho\left(\mathbf{v} \cdot \mathbf{n}_{\phi}\right) \mathbf{n}_{\phi} \\
\rho \frac{\partial e}{\partial t} & +(1-\chi)(\rho \mathbf{v} \cdot \nabla e+P \boldsymbol{\nabla} \cdot \mathbf{v})=-\frac{\chi}{\eta_{c}}\left(\rho \mathbf{n}_{\phi} \cdot \nabla e-\kappa \frac{\rho}{\gamma}\|\mathbf{v}\|^{2}\right)
\end{aligned}
$$




\subsubsection{Penalization of the Conservative Variables}

By transferring to the conservative form, the equations including the penalization terms become:

$$
\begin{aligned}
\frac{\partial \rho}{\partial t} & +(1-\chi) \boldsymbol{\nabla} \cdot(\rho \mathbf{v})=-\frac{\chi}{\eta_{c}}\left(\mathbf{n}_{\phi} \cdot \nabla \rho-\kappa \frac{\rho^{2}}{\gamma P}\|\mathbf{v}\|^{2}\right) \\
\frac{\partial \rho \mathbf{v}}{\partial t}+ & (1-\chi) \boldsymbol{\nabla} \cdot(\rho \mathbf{v} \otimes \mathbf{v}+P \mathbf{I})=-\frac{\chi}{\eta} \rho\left(\mathbf{v} \cdot \mathbf{n}_{\phi}\right) \mathbf{n}_{\phi} \\
& -\frac{\chi}{\eta_{c}}\left(\mathbf{n}_{\phi} \cdot \nabla(\rho \mathbf{v})+\kappa \rho \mathbf{v}\left(1-\frac{\rho}{\gamma P}\|\mathbf{v}\|^{2}\right)\right) \\
\frac{\partial \rho E}{\partial t}+ & (1-\chi) \boldsymbol{\nabla} \cdot((\rho E+P) \mathbf{v})=-\frac{\chi}{\eta} \rho\left(\mathbf{v} \cdot \mathbf{n}_{\phi}\right)^{2} \\
& -\frac{\chi}{\eta_{c}}\left(\rho \mathbf{n}_{\phi} \cdot \nabla E+\frac{(\gamma-1)}{\gamma} \rho \kappa\|\mathbf{v}\|^{2}\right)
\end{aligned}
$$

The energy equation can also be written in terms of total enthalpy by expanding the term containing the curvature and using Eq. (32) and Eq. (39):

$$
\frac{(\gamma-1)}{\gamma} \rho \kappa\|\mathbf{v}\|^{2}=\rho n_{\phi} \cdot \nabla\left(\frac{P}{\rho}\right)
$$

By substitution of Eq. (53) in the energy equation Eq. (52), it gives:

$$
\begin{aligned}
\frac{\partial \rho E}{\partial t} & +(1-\chi) \boldsymbol{\nabla} \cdot((\rho E+P) \mathbf{v})=-\frac{\chi}{\eta} \rho\left(\mathbf{v} \cdot \mathbf{n}_{\phi}\right)^{2} \\
& -\frac{\chi}{\eta_{c}} \rho \mathbf{n}_{\phi} \cdot \boldsymbol{\nabla} H
\end{aligned}
$$

Using this set of penalization terms ensure Eqs. (33)- 36 are respected. Furthermore, the term $\mathcal{P}_{\mathbf{v}}$ replace the dissipation term employed in [13] on the momentum equation. This convection term is derived from physical arguments in order to respect the conservation of total enthalpy instead of using a numerical artifice. Ultimately, this should translate into a reduction in entropy generation, improved conservation of enthalpy and reduced flow separation as shown by [16, 21, 20] with the CCST.

\subsubsection{Implementation Details}

The penalized Euler equations of Eq. (52) are implemented in a cell-centered Finite Volume framework using unstructured meshes. They are written in vector form as:

$$
\frac{\partial \mathbf{W}}{\partial t}+(1-\chi) \boldsymbol{\nabla} \cdot \mathbf{F}_{\text {Euler }}=-\chi \boldsymbol{\nabla} \cdot \mathbf{F}_{i b m}+\chi \mathbf{S}_{i b m}
$$


where the penalization terms are conveniently split into flux $\left(\mathbf{F}_{i b m}\right)$ and source $\left(\mathbf{S}_{i b m}\right)$ terms.

$$
\begin{gathered}
\mathbf{W}=\left[\begin{array}{c}
\rho \\
\rho u \\
\rho v \\
\rho E
\end{array}\right], \quad \mathbf{F}_{\text {Euler }}=\left[\begin{array}{c}
\rho \mathbf{v} \\
\rho u \mathbf{v}+P_{x} \\
\rho v \mathbf{v}+P_{y} \\
\rho H \mathbf{v}
\end{array}\right] \\
\mathbf{W}_{i b m}=\left[\begin{array}{c}
\rho \\
\rho u \\
\rho v \\
\rho H
\end{array}\right], \quad \mathbf{F}_{i b m}=\frac{1}{\eta_{c}}\left[\begin{array}{c}
\rho \mathbf{n}_{\phi} \\
\rho u \mathbf{n}_{\phi} \\
\rho v \mathbf{n}_{\phi} \\
\rho H \mathbf{n}_{\phi}
\end{array}\right]
\end{gathered}
$$

The IB source term $\left(\mathbf{S}_{i b m}\right)$ is split in Dirichlet, convective and curvature contributions:

$$
\begin{gathered}
\mathbf{S}_{i b m}=\mathbf{S}_{i b m, D}+\mathbf{S}_{i b m, \text { conv }}+\mathbf{S}_{i b m, c u r v} \\
\mathbf{S}_{i b m, D}=\frac{1}{\eta}\left[\begin{array}{c}
0 \\
-\rho\left(\mathbf{v} \cdot \mathbf{n}_{\phi}\right) n_{\phi, x} \\
-\rho\left(\mathbf{v} \cdot \mathbf{n}_{\phi}\right) n_{\phi, y} \\
-\rho\left(\mathbf{v} \cdot \mathbf{n}_{\phi}\right)^{2}
\end{array}\right] \\
\mathbf{S}_{i b m, c o n v}=\frac{1}{\eta_{c}}\left[\begin{array}{c}
\rho \boldsymbol{\nabla} \cdot \mathbf{n}_{\phi} \\
\rho u \boldsymbol{\nabla} \cdot \mathbf{n}_{\phi} \\
\rho v \boldsymbol{\nabla} \cdot \mathbf{n}_{\phi} \\
\rho H \nabla \cdot \mathbf{n}_{\phi}
\end{array}\right] \\
\mathbf{S}_{i b m, \text { curv }}=\frac{1}{\eta_{c}} \kappa\left[\begin{array}{c}
\rho \frac{\rho}{\gamma P}\|\mathbf{v}\|^{2} \\
\rho u\left(\frac{\rho}{\gamma P}\|\mathbf{v}\|^{2}-1\right) \\
\rho v\left(\frac{\rho}{\gamma P}\|\mathbf{v}\|^{2}-1\right) \\
\rho H \frac{\rho}{\gamma P}\|\mathbf{v}\|^{2}
\end{array}\right]
\end{gathered}
$$

where the convective source term $\mathbf{S}_{i b m, c o n v}$ comes from rewriting Eq. 52 to isolate $\mathbf{F}_{i b m}$. From the IB flux (Eq. (57)), the system is shown to be hyperbolic in the solid with eigenvalues $\mathbf{n}_{\phi}, \mathbf{n}_{\phi}, \mathbf{n}_{\phi}$ and $\gamma \mathbf{n}_{\phi}$. 
The penalization of the Euler equations leads to a stiff system as the penalization parameters $\left(\eta\right.$ and $\eta_{c}$ ) are very small. One way to alleviate this problem is to solve the system implicitly. In this paper it is solved using a BICGSTAB algorithm with a block Jacobi preconditioner. The Euler fluxes are computed using a Roe scheme [25] (no entropy fix) and a MUSCL reconstruction to achieve $2^{\text {nd }}$ order accuracy in space [26]. The IB fluxes are evaluated with a simple $1^{\text {st }}$ order upwind scheme (upwinded by $\mathbf{n}_{\phi}$ ) of the form:

$$
\begin{aligned}
\mathbf{F}_{\text {edge }} & =\frac{1}{2}\left[\left(\mathbf{n}_{\phi} \cdot \mathbf{n}_{\text {edge }}\right)\left(\mathbf{W}_{i b m, R}+\mathbf{W}_{i b m, L}\right)\right. \\
& \left.-\left|\mathbf{n}_{\phi} \cdot \mathbf{n}_{\text {edge }}\right|\left(\mathbf{W}_{i b m, R}-\mathbf{W}_{i b m, L}\right)\right]
\end{aligned}
$$

where $L$ and $R$ represent the left and right state respectively and $\mathbf{n}_{e d g e}$ is the normal vector to the cell edge.

The mask function $\chi$ is the Heaviside function $(\mathcal{H})$ based on the signed distance field $\phi$.

$$
\chi=\mathcal{H}(-\phi)
$$

The level-set $\phi=0$ determines the location of the IB with $\phi<0$ in the solid and $\phi>0$ in the fluid. It can be seen that in the fluid $(\chi=0)$, the usual Euler equations are retrieved while in the solid $(\chi=1)$ only the penalization terms are activated.

From [13, $\eta_{c} \ll 1$ and $\eta \ll 1$ in order to enforce the correct condition in the solid by penalization. In the current implementation, a slightly different approach is followed. Since the Euler fluxes are deactivated in the solid, $\eta_{c}$ is not required to be very small in order to propagate the fluid properties towards the solid. Here, the IB fluxes are not penalized but are merely a replacement for the Euler fluxes inside the solid by using $\eta_{c}=1$. It comes back to solving propagation equations in the solid, which are penalized to apply the no-penetration 
velocity. By using $\eta_{c}=1$, the equations in the solid are:

$$
\begin{aligned}
& \frac{\partial \rho}{\partial t}+\mathbf{n}_{\phi} \cdot \nabla \rho=\kappa \frac{\rho^{2}}{\gamma P}\|\mathbf{v}\|^{2} \\
& \frac{\partial \rho \mathbf{v}}{\partial t}+\mathbf{n}_{\phi} \cdot \nabla(\rho \mathbf{v})=\kappa \rho \mathbf{v}\left(\frac{\rho}{\gamma P}\|\mathbf{v}\|^{2}-1\right)-\frac{1}{\eta} \rho\left(\mathbf{v} \cdot \mathbf{n}_{\phi}\right) \mathbf{n}_{\phi} \\
& \frac{\partial \rho E}{\partial t}+\rho \mathbf{n}_{\phi} \cdot \nabla H=-\frac{1}{\eta} \rho\left(\mathbf{v} \cdot \mathbf{n}_{\phi}\right)^{2}
\end{aligned}
$$

where the only penalization terms are $\frac{1}{\eta} \rho\left(\mathbf{v} \cdot \mathbf{n}_{\phi}\right) \mathbf{n}_{\phi}$ and $\frac{1}{\eta} \rho\left(\mathbf{v} \cdot \mathbf{n}_{\phi}\right)^{2}$. A typical value for the penalization parameter is $\eta=10^{-10}$, which accurately enforces the slip velocity $\left(\mathbf{v} \cdot \mathbf{n}_{\phi}=0\right)$ near the IB. As for the volume penalization method, $\mathbf{v} \cdot \mathbf{n}_{\phi}$ exponentially tends towards zero (almost instantaneously). This approach is suitable if the Euler equations are solved for a steady state.

In this paper $\eta_{c}=1$ is used, but it is also possible to use $\eta_{c}<1$. In such a case, it is important to respect a ratio $\eta / \eta_{c}$ as there is an interaction between the Neumann and Dirichlet conditions in the momentum equation. The idea is to keep the imposition of $\mathbf{v} \cdot \mathbf{n}=0$ dominant over the propagation of the information. To do so $\eta \ll \eta_{c}$ and a ratio of $\eta / \eta_{c}=10^{-6}$ was found to be sufficient for most applications.

With the CBVP-Hs method, two adjustable parameters are used: $\eta$ and $\eta_{c}$. Thus, it has fewer parameters to calibrate compared to the CBVP method which has three of them: $\eta, \eta_{c}$ and $\nu_{\eta}$.

\subsubsection{Dimension of the Problem}

The approach was derived and assessed in 2D in the present article, without losing generality. Since the penalization method is based on the continuous form of the equations and is independent of the discretization, the CBVP-Hs can naturally be extended to 3D. In practice, some difficulties might arise when evaluating the curvature term $(\kappa)$, a key element of the method. In $2 \mathrm{D}$, the curvature is purely geometric and therefore simple to estimate. For 3D applications, the curvature also depends on the direction of the wall streamlines and is therefore linked to the flow velocity at the wall. This additional difficulty is covered for instance in [15] and [20, where the CBVP method and a 3D im- 
plementation of the ghost cell method are respectively discussed. Thus a 3D implementation of the CBVP-Hs method should be straightforward by building on previous work from the literature.

\subsection{Notes on Moving Boundaries}

Although moving boundaries are not considered in this paper, the CBVP-Hs method is still directly applicable where boundary displacement can be decoupled from the aerodynamic flow. For instance, in typical numerical tools for the prediction of in-flight icing (e.g. LEWICE [17, IGLOO2D [18]), the aerodynamic flow is computed to steady state and then the ice shape is updated according to the ice growth in a segregated step. With the current penalization method, the geometry update can be accounted for in the aerodynamic solver by re-evaluating the signed distance field $(\phi)$ and associated metrics $\left(\mathbf{n}_{\phi}, \kappa\right.$, $\chi$ ). By doing so, the penalization method automatically applies the boundary conditions on the new geometry. Note that the CBVP-Hs method is not limited to icing applications. A similar process could be applied to perform shape optimization where a steady or unsteady flow is computed on a fixed geometry. The shape update would again be accounted for by re-evaluating the signed distance field.

\subsection{Geometry Fidelity}

The volume penalization method is limited to $1^{\text {st }}$ order accuracy because the boundary condition is applied at the cell centers close to the boundary and not on the immersed boundary itself. Some extensions to second order accuracy are available in the literature [27, 28] by using information at the discrete level. Here the implementation is limited to the classical first order accuracy. With this type of approach, the accuracy of the method is usually improved by refining the mesh in the vicinity of the IB. 


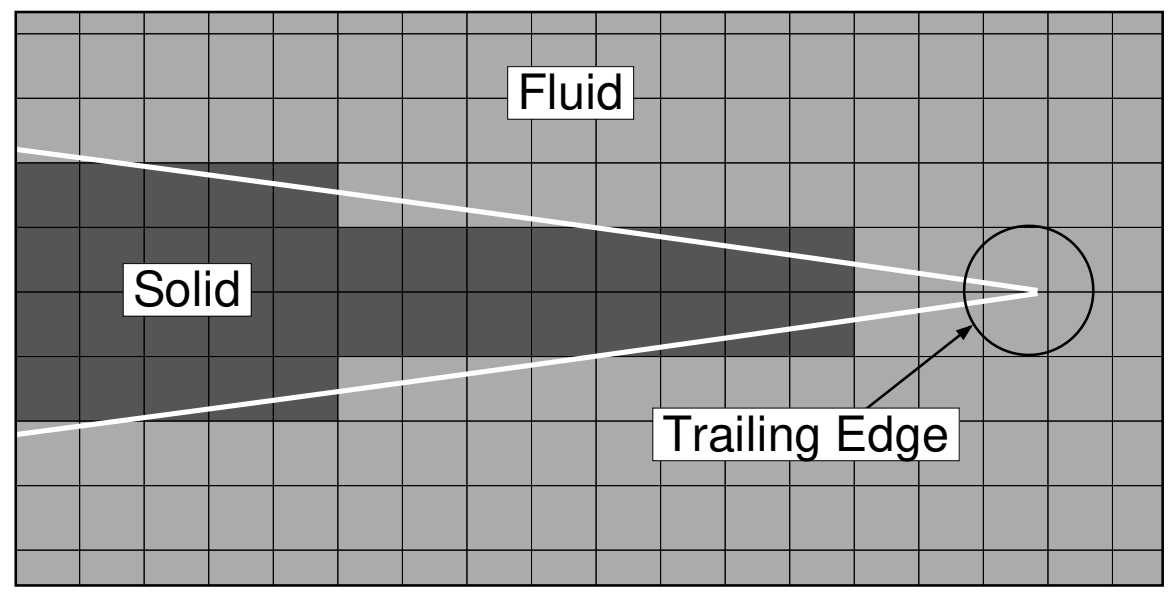

Figure 3: Trailing edge representation for a NACA0012 airfoil (white line) on a Cartesian grid

In the current implementation, even if the sharp Heaviside function leads to a staircase representation of the IB, the Neumann boundary conditions (convective terms) are smoothing the interface and improving the solution on coarser meshes (e.g. when extracting the pressure coefficients). However, because of the staircase representation of the IB and because the cell centers are used to determine if a cell is fluid or solid, the current implementation is not well suited to deal with sharp features (e.g. sharp trailing edge of an airfoil). As shown on Figure 3, the sharp trailing edge is seen as blunt by the penalization method as the fluid cells covering the trailing edge are not penalized. A refinement in the vicinity of the sharp feature can improve the solution by providing a more accurate representation of the geometry. In this paper, the sharp trailing edge is simply treated as blunt. In this way, the cell refinement can be coarser while allowing the correct overall solution to be retrieved. This approach is used for the NACA0012 airfoil presented in the next section.

For icing applications [29], the selected approach is to deal with sharp features on the clean geometry using a body-fitted mesh (e.g. sharp trailing edge on an airfoil). Only the ice will be immersed in the mesh (see Figure 18b. Although some sharp features may be generated by the ice growth, it is acceptable for them to be slightly smoothed out. With this approach, the accuracy of the 
body-fitted approach is retrieved where possible and the flexibility of the IBM is used otherwise. The ice accretion case presented in this paper follows this approach.

\subsection{Mesh Particularities}

For unstructured meshes made of triangles, some cell configurations lead to a poor propagation of the information from the fluid to the solid. This situation occurs when a fluid cell near the IB is trapped between solid cells and the numerical fluxes allow no communication with neighboring fluid cells. One example is illustrated in Figure 4, where cell A is fluid and cell B is solid. Cell A has 2 solid cells and one fluid cell as direct face neighbors. In this specific configuration, cell A is emptied and the solution is propagated in the solid (e.g. cell B).

In this paper, the issue is solved by applying a correction on the mask function $(\chi)$ for these pathological cells: instead of using a sharp definition $(\chi=0$ or 1$), \chi$ is set to the solid fraction of the cell $(\chi \in[0,1])$. This correction blends the Euler and the penalization fluxes leading to a smoother solution as the communication with the fluid is restored. 


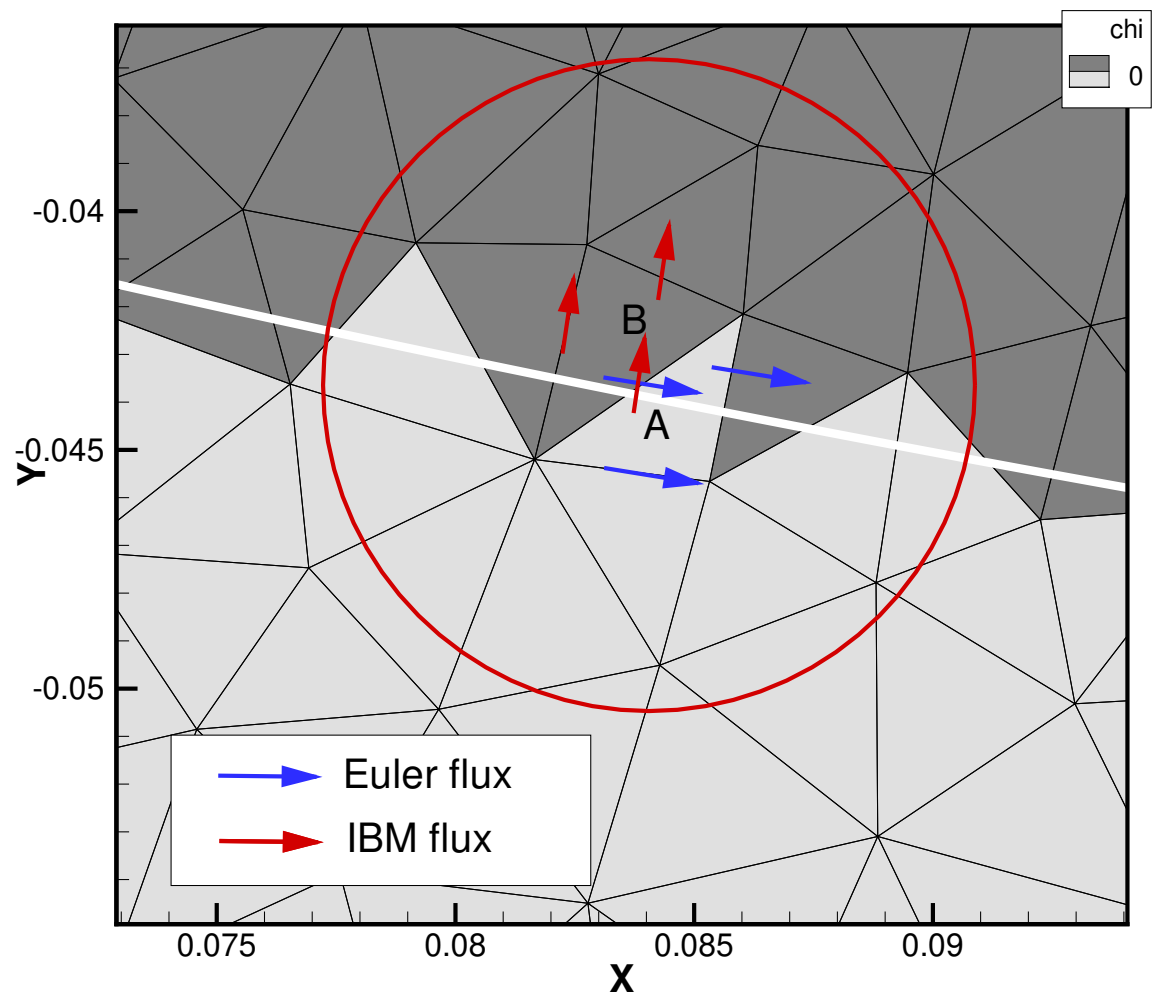

Figure 4: Pathological cell example with flux representation and mask function $\chi$

From numerical experiments, the issue was observed for only a few cells (1\% to $2 \%$ of the IB cells) and not for all meshes. Some unstructured meshes will present no pathological cells while for structured meshes made of quadrilateral cells, the issue was never observed. Also, note that the issue is present for both the CBVP and CBVP-Hs methods.

\subsection{Brief Comparison with other type of IBMs}

For completion, please note that other type of IBMs have been successfully applied to the Euler equations, offering their own set of advantages and drawbacks. For discrete approaches, the desired boundary conditions are imposed by enforcing them at the discrete level (e.g. [21, 20, [19]). Thus they are dependent on the selected discretization method and also on the type of mesh used. These approaches have the advantage of being accurate ( $2^{\text {nd }}$ order, e.g. [30]) 
and can also deal with sharp trailing edges in a simple manner (e.g. [21, 19]). Embedded "cut-cell" methods (e.g. [31, 32]) are also suitable for solving the Euler equations with similar advantages compared to discrete methods. This paper inserts itself in the scope of penalization methods and suggests a suitable approach for the penalization of the Euler equations. Although the current CBVP-Hs method is only $1^{\text {st }}$ order accurate (globally), the advantage of penalization methods lies in their independence from the discretization method or the type of mesh used as they are based on the continuous form of the equations. They are also independent of the physical dimension of the problem and simple to implement as they require minimal information from the geometry. A simple evaluation of the signed distance field $(\phi)$ and mask function $(\chi)$ is sufficient to determine the location of the immersed boundary and activate the penalization terms in the solid.

\section{Results}

In this section, the behavior of the CBVP-Hs method is shown on different test cases. A mesh refinement study is first performed for the weakly compressible flow around a circular cylinder. Then the subsonic flow around a NACA0012 airfoil is studied. The penalization method is also tested on an ice horn which was found difficult to solve in a previous communication [29] due to its high curvature. The simulation parameters are summarized in Table 1.

The solution from the penalization method is verified against analytical solutions when applicable or against numerical solutions obtained by a body-fitted approach. A comparison is also performed against the CBVP method. Wall pressure coefficients, entropy and total enthalpy errors are compared and discussed. 
Table 1: Simulation Parameters

\begin{tabular}{l|ccc} 
& Cylinder & Airfoil & Ice Horn \\
\hline Geometry & Cylinder & NACA0012 & GLC305 \\
Chord & $\mathrm{D}=2.0$ & 1.0 & 0.9144 \\
AoA & 0.0 & 1.25 & 4.0 \\
Mach & 0.1 & 0.5 & 0.273 \\
$\mathbf{P}_{\text {static }}$ & $100 \mathrm{kPa}$ & $100 \mathrm{kPa}$ & $101.325 \mathrm{kPa}$ \\
$\mathbf{T}_{\text {static }}$ & $300.0 \mathrm{~K}$ & $300.0 \mathrm{~K}$ & $268.3 \mathrm{~K}$ \\
LWC & - & - & $0.54 \mathrm{~g} / \mathrm{m}^{3}$ \\
MVD & - & - & $20 \mu \mathrm{m}$ \\
Icing Time & - & - & $1350 \mathrm{~s}$ \\
\hline
\end{tabular}

In Table 1, LWC stands for Liquid Water Content and represents the mass of water per volume of air. The median volume diameter (MVD) represents the droplet size for the icing simulation. The list of icing parameters are provided but note that only the aerodynamics is treated in this paper.

For the following simulations, the convergence is determined by monitoring the $L_{2}$ and $L_{\infty}$ norms of the wall pressure coefficient $(C p)$. The usual density, momentum and energy residuals are also monitored. In the following sections, when the convergence threshold is specified, the criterion on $C p$ is used. For the penalization method, the convergence check requires a pressure interpolation at the IB at each time step. It is performed by a weighted least square interpolation method.

\subsection{Weakly Compressible Flow Around a Cylinder}

The first test case is the weakly compressible flow around a circular cylinder. As the Euler flow solver in IGLOO2D is compressible only, the incompressible analytical solution is approached by performing the simulation at a low Mach number $($ Mach $=0.1)$. The analytical solution for the pressure coefficient $(C p)$ 
is, in 2D Cartesian coordinates:

$$
C p_{\text {analytical }}=\frac{2 R_{c}^{2}\left(x^{2}-y^{2}\right)-R_{c}^{4}}{\left(x^{2}+y^{2}\right)^{2}}
$$

where $R_{c}$ is the radius of the cylinder.

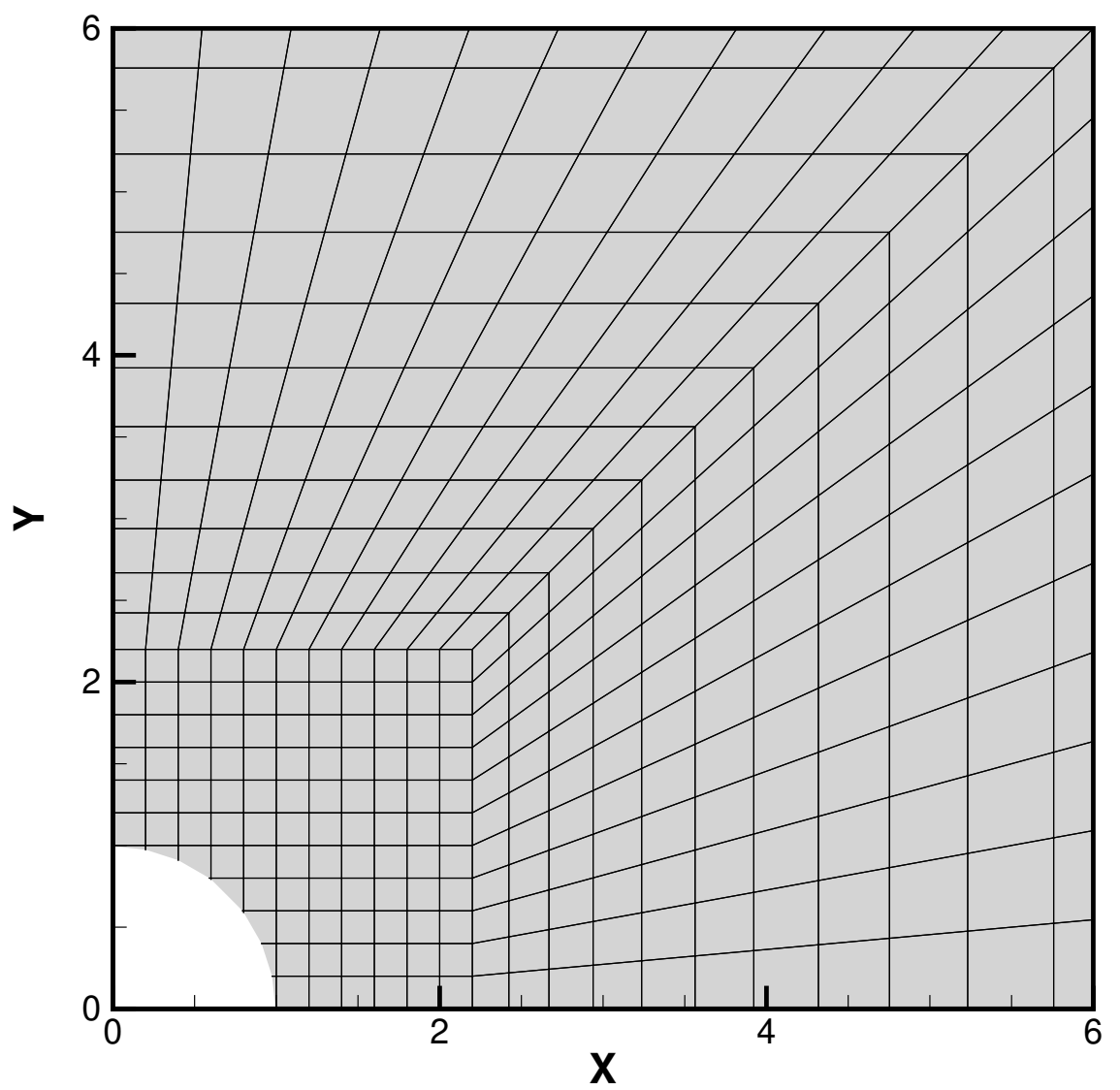

Figure 5: Mesh for the cylinder with cell size $D / \Delta x=10$

A family of five meshes is generated ranging from $D / \Delta x=10$ to $D / \Delta x=$ 160 , where $D$ is the diameter of the cylinder and $\Delta x$ the Cartesian cell size. The cylinder is immersed into a uniform Cartesian grid. To reduce the total number of cells the uniform Cartesian grid is limited to a width of 2 diameters. Outside this zone a structured mesh is used allowing cell growth and stretching, 
but still ensuring mesh symmetry. The far field is located at 50 diameters from the cylinder. Part of the mesh for $D / \Delta x=10$ is illustrated in Figure 5 with a zoom on the blanked cylinder.

For the results presented below, the wall $\mathrm{Cp}$ residual is converged to $10^{-8}$. Figures 6 and 7 illustrate the $C p$ distribution for both the CBVP and CBVPHs methods. From these figures, both methods converge towards the analytical solution. However, the mesh convergence is faster for the CBVP-Hs method for which a good global $C p$ distribution is achieved on a mesh as coarse as $D / \Delta x=40$. In comparison, the CBVP method exhibits a satisfactory global solution for the finest mesh only $(D / \Delta x=160)$.

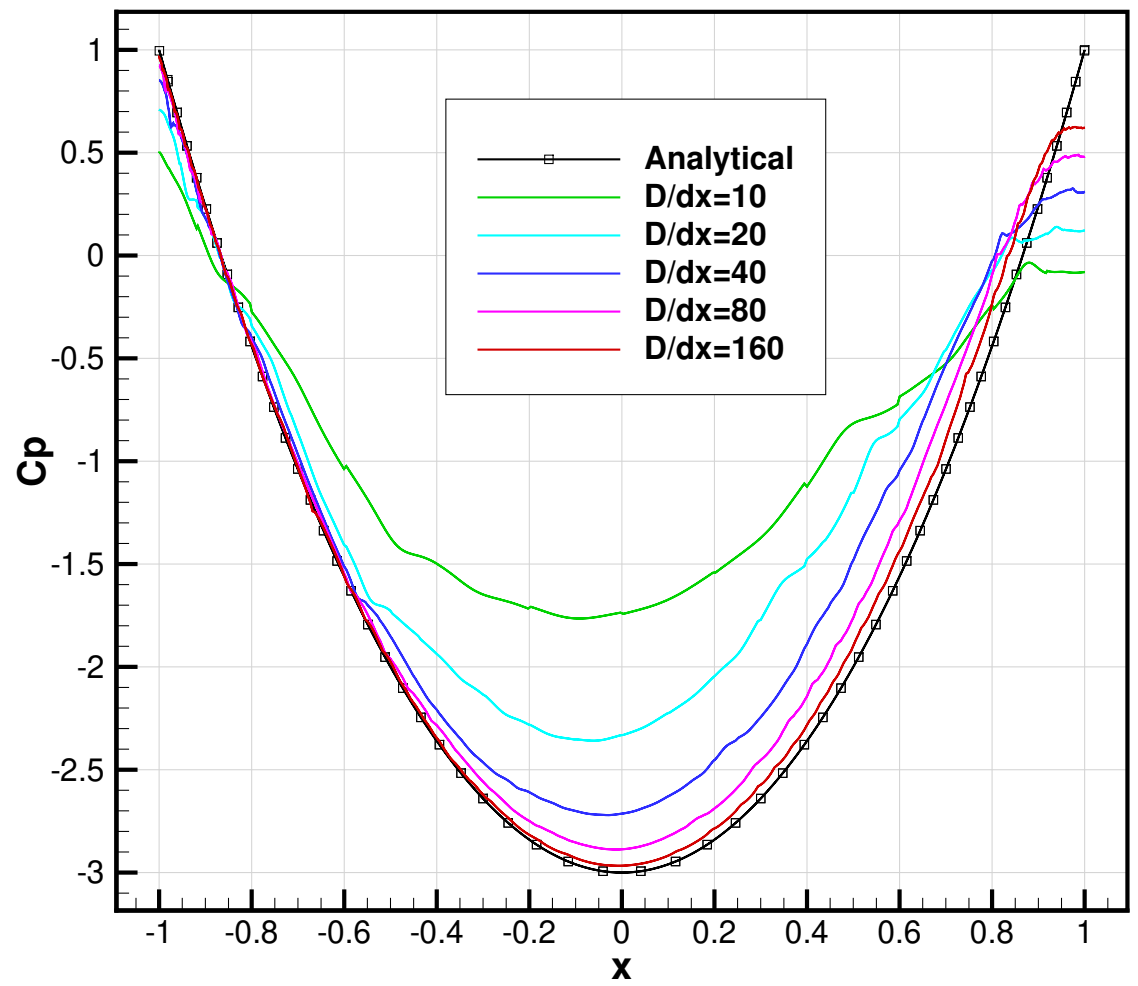

Figure 6: Wall Cp with mesh refinement for the cylinder (CBVP) 


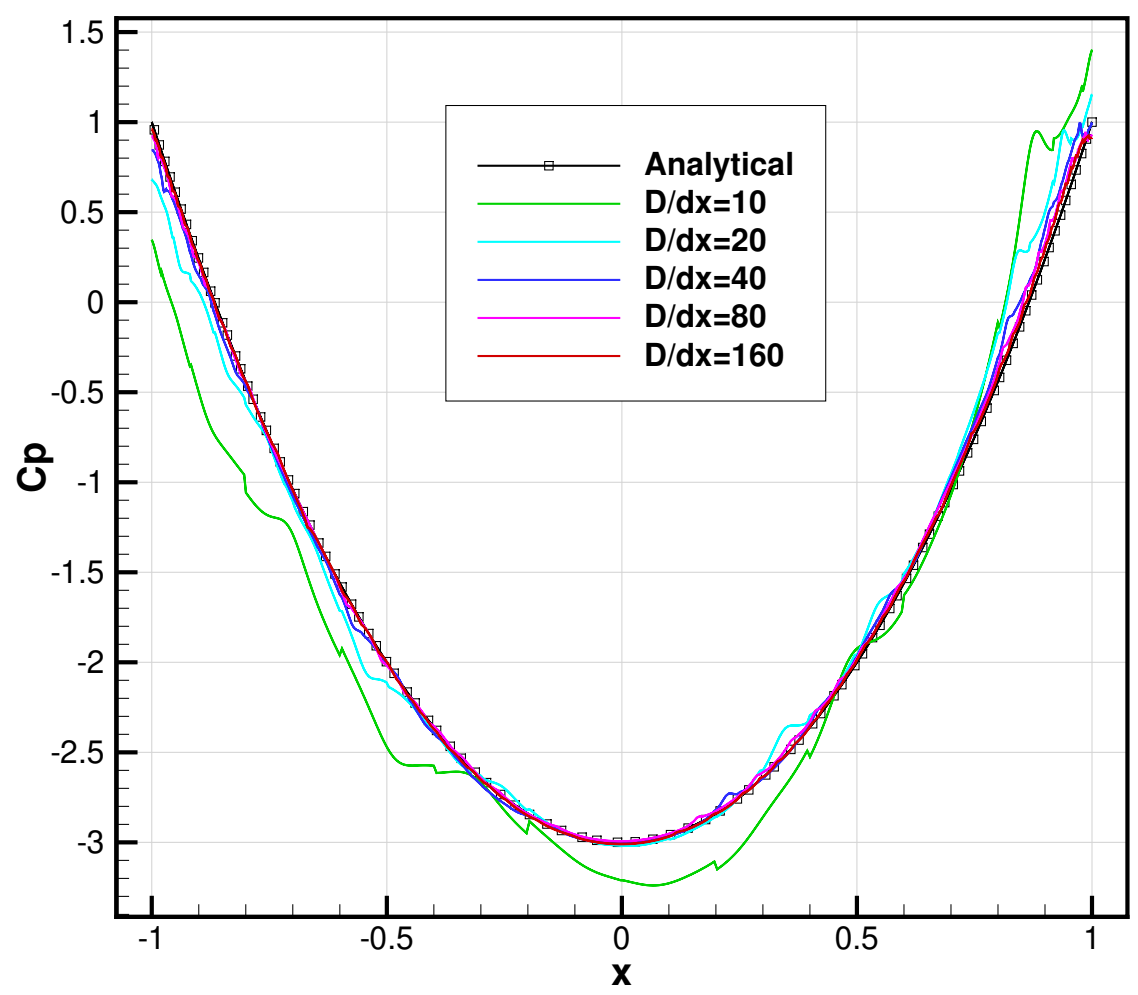

Figure 7: Wall Cp with mesh refinement for the cylinder (CBVP-Hs)

For a more detailed analysis, pressure coefficients are extracted at locations of interest: $(x, y)=(-1,0),(x, y)=(0,1)$ and $(x, y)=(1,0)$. For these locations the expected analytical solution is respectively: $C p=1, C p=-3$ and $C p=1$. The data is shown on Figure 8 as the Cp error $(\Delta C p)$ against the analytical solution for the three locations:

$$
|\Delta C p|=\left|C p-C p_{\text {analytical }}\right|
$$

The observation of Figure 8 shows that as the mesh is refined the error for the forward stagnation point $(x=-1)$ is roughly the same for both methods (CBVP and CBVP-Hs). However, the error is much lower at the maximum suction peak $(x=0)$ and at the aft stagnation point $(x=1)$ for the CBVP-Hs method. For instance, on a mesh as coarse as $D / \Delta x=20$ the suction peak is captured to 
an accuracy of $\Delta C p \approx 10^{-4}$ with the CBVP-Hs compared to $\Delta C p \approx 10^{-0.5}$ for the CBVP method. The reattachment point at $x=1$ is also captured more accurately with $\Delta C p \approx 10^{-2.5}$ for the CBVP-Hs against $\Delta C p \approx 10^{-1}$ on the finest mesh $(D / \Delta x=160)$. For the forward stagnation point, the order of convergence is observed to be 1 for both methods. It is, however, difficult to conclude on the order of convergence for the other locations. The order of convergence will be discussed based on the root mean squared (RMS) in the next section.

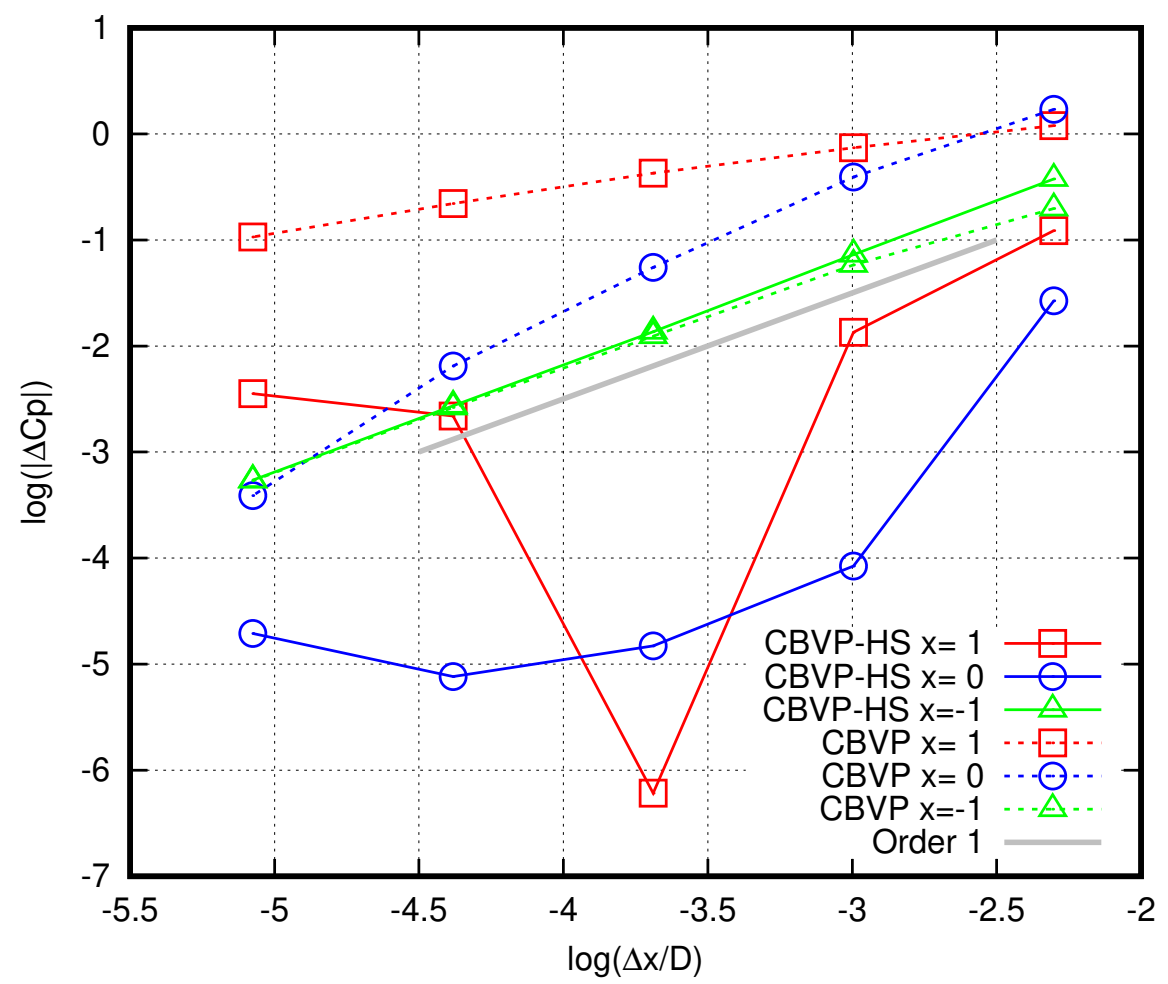

Figure 8: Cylinder $\Delta C p$ convergence as the mesh is refined

\subsubsection{Entropy and Total Enthalpy}

As the flow around the cylinder presents no heat source nor external force, the entropy $(\mathrm{s})$ and total enthalpy $(\mathrm{H})$ should be conserved in the entire computational domain. Thus, entropy and total enthalpy errors can be evaluated 
against the freestream conditions as:

$$
\begin{aligned}
\Delta s & =\left|\left(s-s_{\infty}\right)\right| / s_{\infty} \\
\Delta H & =\left|\left(H-H_{\infty}\right)\right| / H_{\infty} \\
s_{\infty} & =P_{\infty} / \rho_{\infty}^{\gamma} \\
H_{\infty} & =\frac{\gamma}{\gamma-1} \frac{P_{\infty}}{\rho_{\infty}}+\frac{1}{2}\left\|\mathbf{v}_{\infty}\right\|^{2}
\end{aligned}
$$

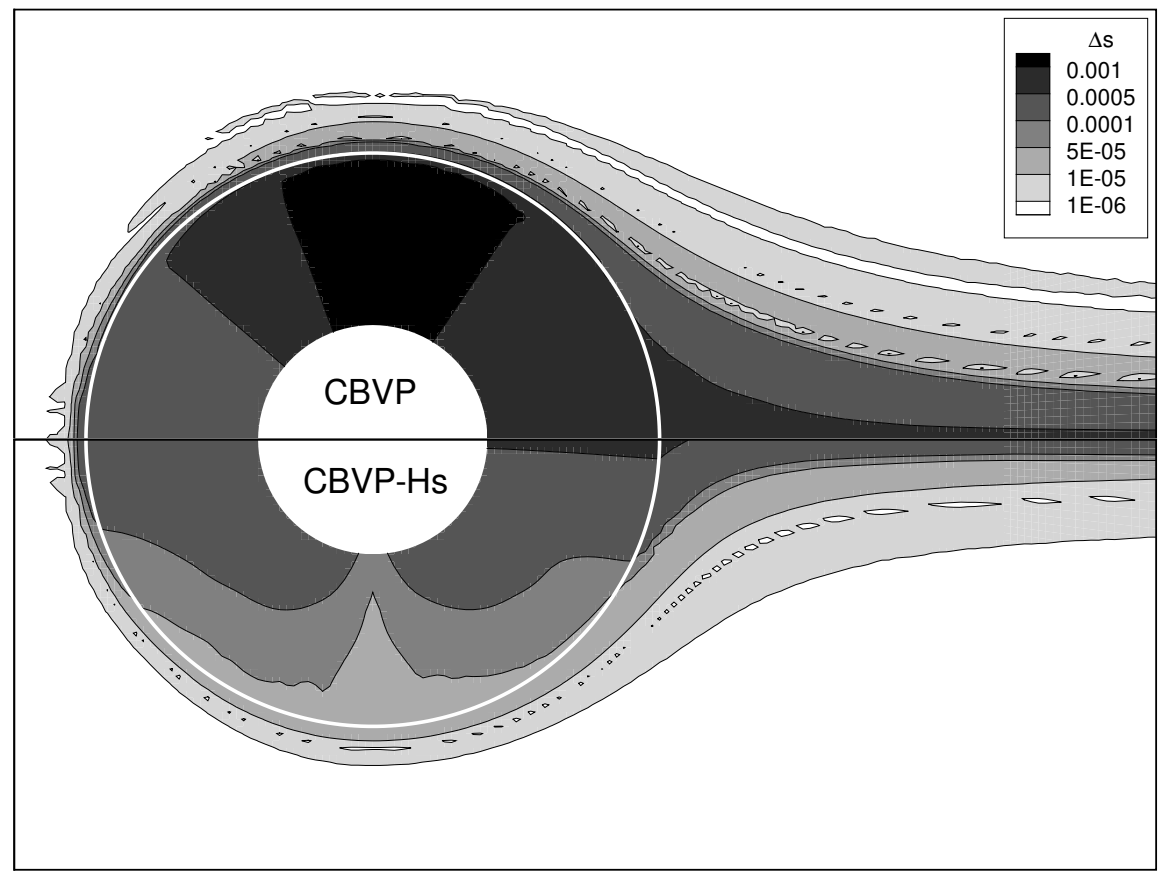

Figure 9: Entropy error for the cylinder on the Cartesian mesh $D / \Delta x=80$. Immersed Boundary: white line. The center of the cylinder is blanked for clarity. 


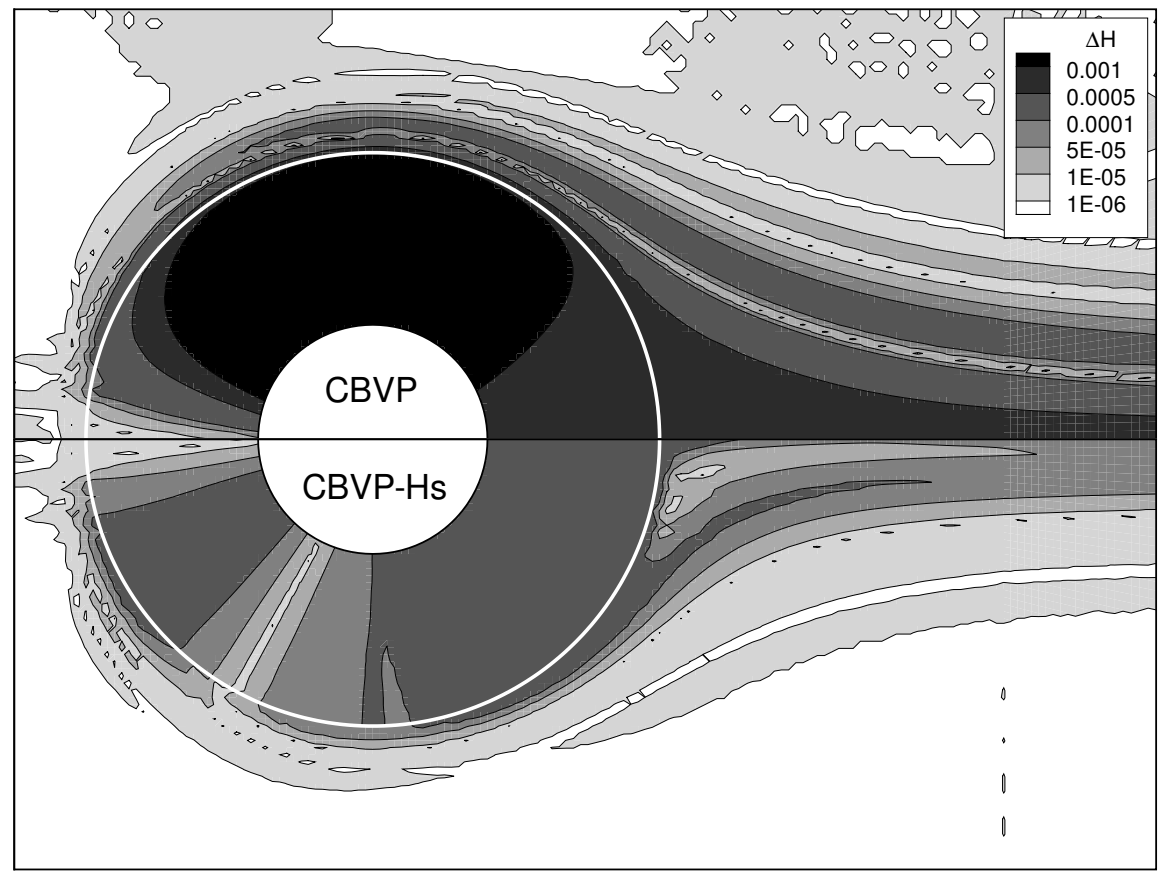

Figure 10: Total enthalpy error for the cylinder on the Cartesian mesh $D / \Delta x=80$. Immersed Boundary: white line. The center of the cylinder is blanked for clarity.

The contours for the entropy and total enthalpy error are illustrated on Figures 910 These figures confirm that the CBVP-Hs method reduces the error in the wake and at the maximum suction points of the cylinder. However, the error is similar for both methods at the forward stagnation point. As an additional comparison point, the maximum entropy error in the field for the CBVP is $\approx 1 \mathrm{e}-3$ while it is $\approx 6 \mathrm{e}-4$ for the CBVP-Hs. The same behavior is observed for the total enthalpy error whose maximum is $\approx 1 \mathrm{e}-3$ for the CBVP and $\approx 3 \mathrm{e}-4$ for the CBVP-Hs. As a comparison, a body-fitted simulation using an equivalent wall cell size was performed (not shown). The error for entropy and total enthalpy is respectively $\approx 1 \mathrm{e}-5$ and $\approx 8 \mathrm{e}-6$ using the CCST boundary condition, which shows that even for a body-fitted mesh the entropy and total enthalpy are not conserved to machine accuracy.

The error at the wall for $C p, s$ and $H$ are plotted on Figure 11 for both 
methods and for the different refinement levels. The error is presented as the RMS of the local $\Delta C p, \Delta s$ and $\Delta H$ respectively. This gives an indication of the global wall error (RMS) and confirms that the CBVP-Hs is more accurate. In fact, the CBVP solution with $D / \Delta x=160$ is equivalent to the CBVP-Hs with $D / \Delta x=40$ in terms of the RMS.

From Figure 11, the error is observed to be lower for the CBVP-Hs method while also offering a faster convergence rate on $\Delta H$ and $\Delta s$. The order of convergence is observed to be 1 for the CBVP-Hs method on entropy and total enthalpy, while it is slightly lower for the CBVP method. The volume penalization method is limited to $1^{\text {st }}$ order accuracy, thus it is logical to obtain a global $1^{\text {st }}$ order of convergence at best although the solution is $2^{\text {nd }}$ order accurate in the field ( $2^{\text {nd }}$ order Roe Scheme).

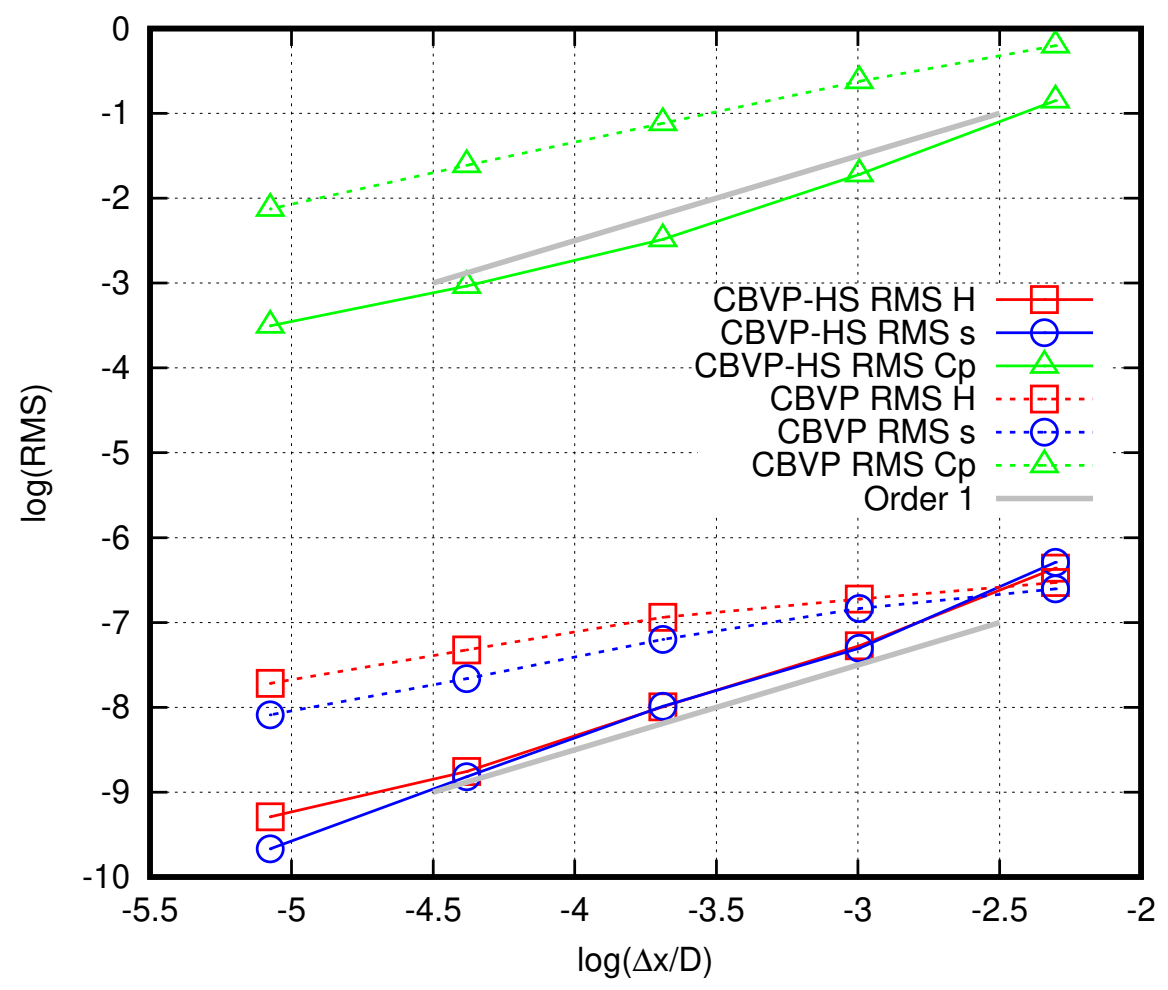

Figure 11: Global wall error (RMS) for the cylinder 


\subsubsection{Continuity of the Solution}

The cylinder case with $D / \Delta x=80$ is used to illustrate the continuity of the solution in the vicinity of the IB and inside the solid zone. Both the CBVP and CBVP-Hs are designed with hyperbolic penalization terms on the continuity and energy equations allowing the propagation of information from the fluid to the solid in the normal direction to the interface. However, the two methods differ on the momentum equation where the continuity of the velocity is imposed by an artificial dissipation term for the CBVP and by a hyperbolic penalization term for the CBVP-Hs. For both methods, a no-penetration velocity $\left(\mathbf{v} \cdot \mathbf{n}_{\phi}=0\right)$ is enforced in the solid, thus only the tangential component of the velocity remains.

As shown on Figure 12 the tangential velocity is continuous across the IB for both methods. For the CBVP, the continuity is enforced via numerical dissipation. This does not only produce an unphysical extension of the tangential velocity in the solid, it also pollutes the solution in the fluid near the IB. On the other hand, the CBVP-Hs extends the tangential velocity following the physics of the wall streamline. Also note that the curvature terms are only activated in the vicinity of the interface, the remainder of the solid zone only sees convection terms in the direction normal to the IB. It translates into a proper extension of the fluid properties near the IB, then into straight contour lines pointing towards the center of the cylinder. 


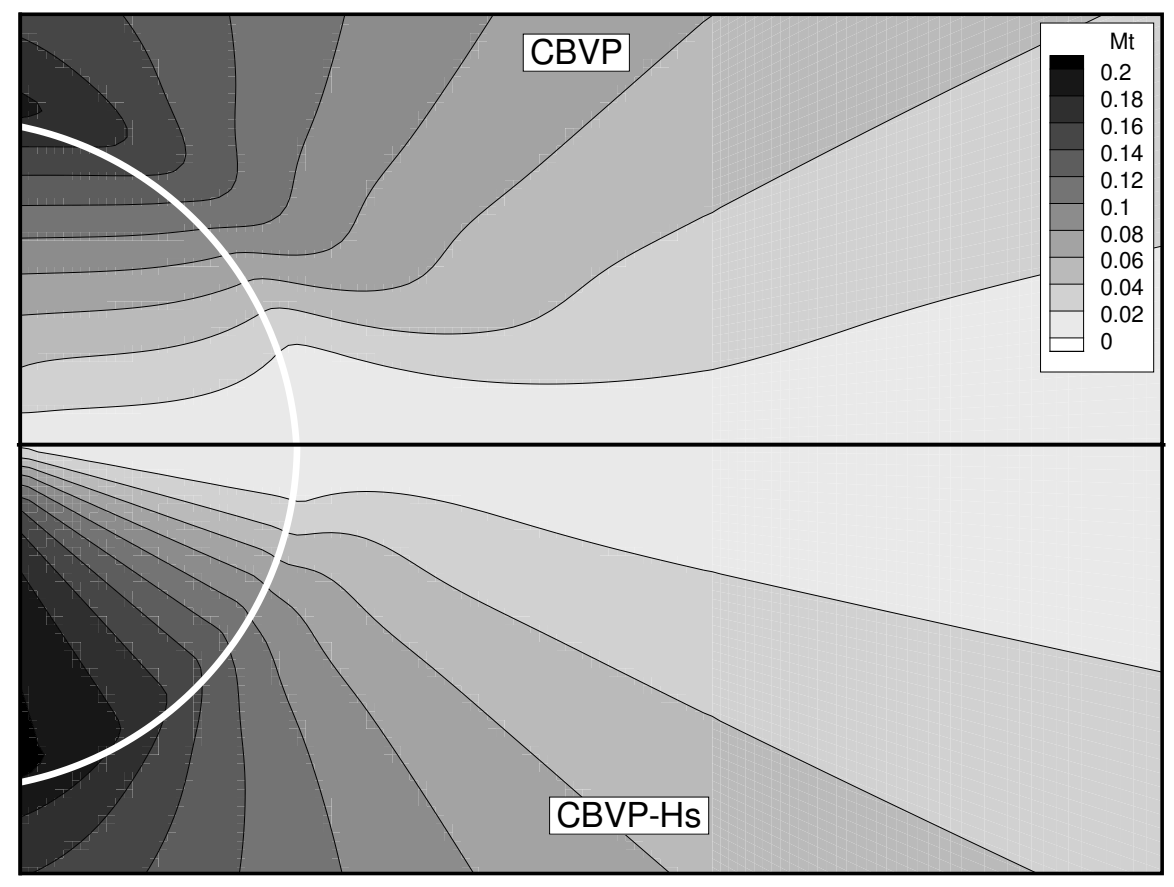

Figure 12: Continuity of the tangential Mach number $\left(M_{t}\right)$ near the IB and inside the solid for both CBVP and CBVP-Hs $(D / \Delta x=80)$.

The continuity of the pressure $(C p)$ is illustrated on Figure 13. In terms of pressure both the CBVP and CBVP-Hs methods behave in a similar and satisfactory manner inside the solid. The main difference is coming from the quality of the solution, which is more accurate for the CBVP-Hs on an equivalent mesh. This is evidenced for instance by the Cp near the aft stagnation point which is closer to the analytical value of $C p=1$. 


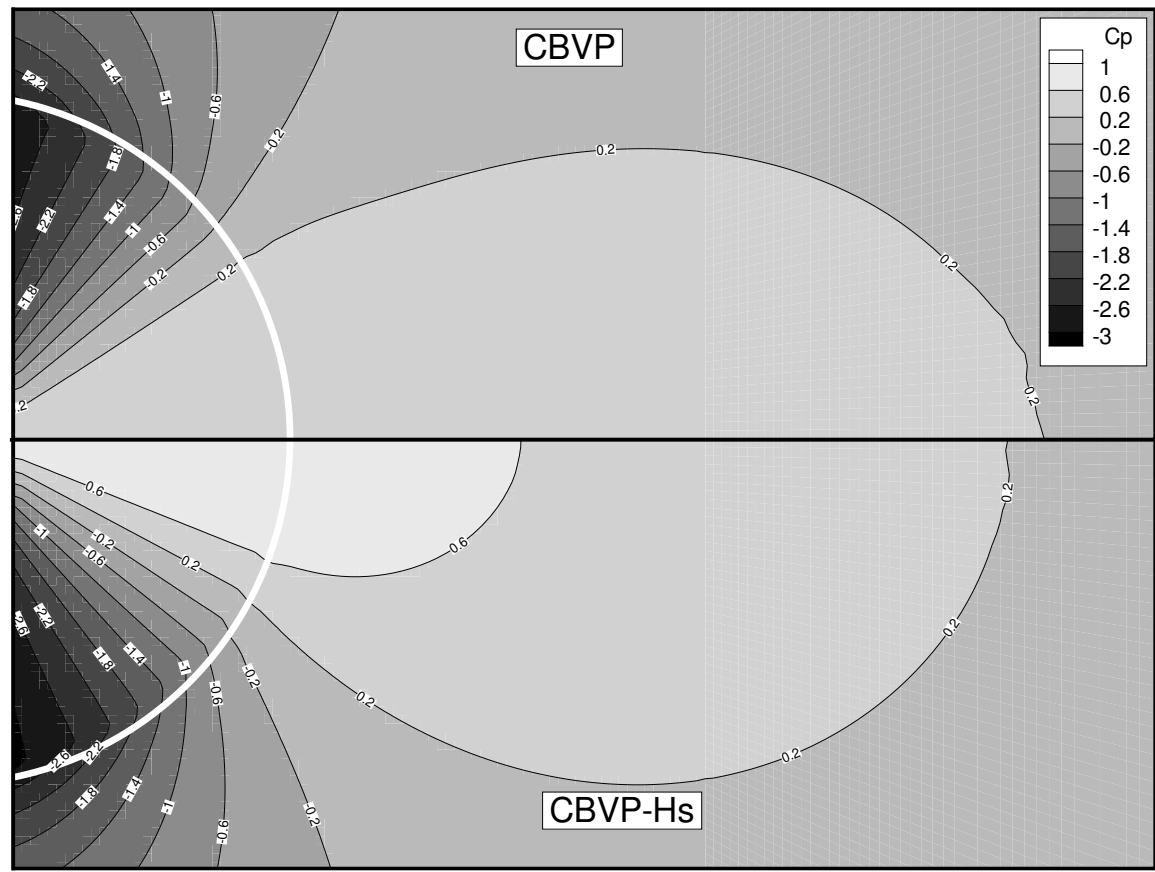

Figure 13: Continuity of the pressure coefficient $(C p)$ near the IB and inside the solid for both CBVP and CBVP-Hs $(D / \Delta x=80)$.

It is also interesting to verify the continuity of entropy and total enthalpy as it should be strongly imposed using the CBVP-Hs. As illustrated in Figures 9 10. both methods propagate the entropy and total enthalpy from the fluid to the solid. The CBVP method exhibits larger error at the suction peak and aft of the cylinder on the fluid side. This error is also propagated into the solid. On the other hand, the CBVP-Hs method shows a lower error on the fluid side (for both $\Delta s$ and $\Delta H)$. This is also reflected inside the solid as expected. These two figures emphasize the difference in accuracy of the two methods in the vicinity of the IB, with a better solution achieved by the CBVP-Hs.

\subsection{Subsonic Flow Around a NACA0012}

A more representative test case for aerospace applications is the subsonic flow around an airfoil. As stated earlier, the first order accurate penalization 
method is not well suited for the representation of sharp features like trailing edges. Here, the issue is worked around by using a NACA0012 airfoil with a blunt trailing edge [33], allowing easier meshing of the solid (interior mesh). An unstructured mesh is used to show that the method is also applicable to general meshes. This also allows a better comparison between the body-fitted and penalization methods.
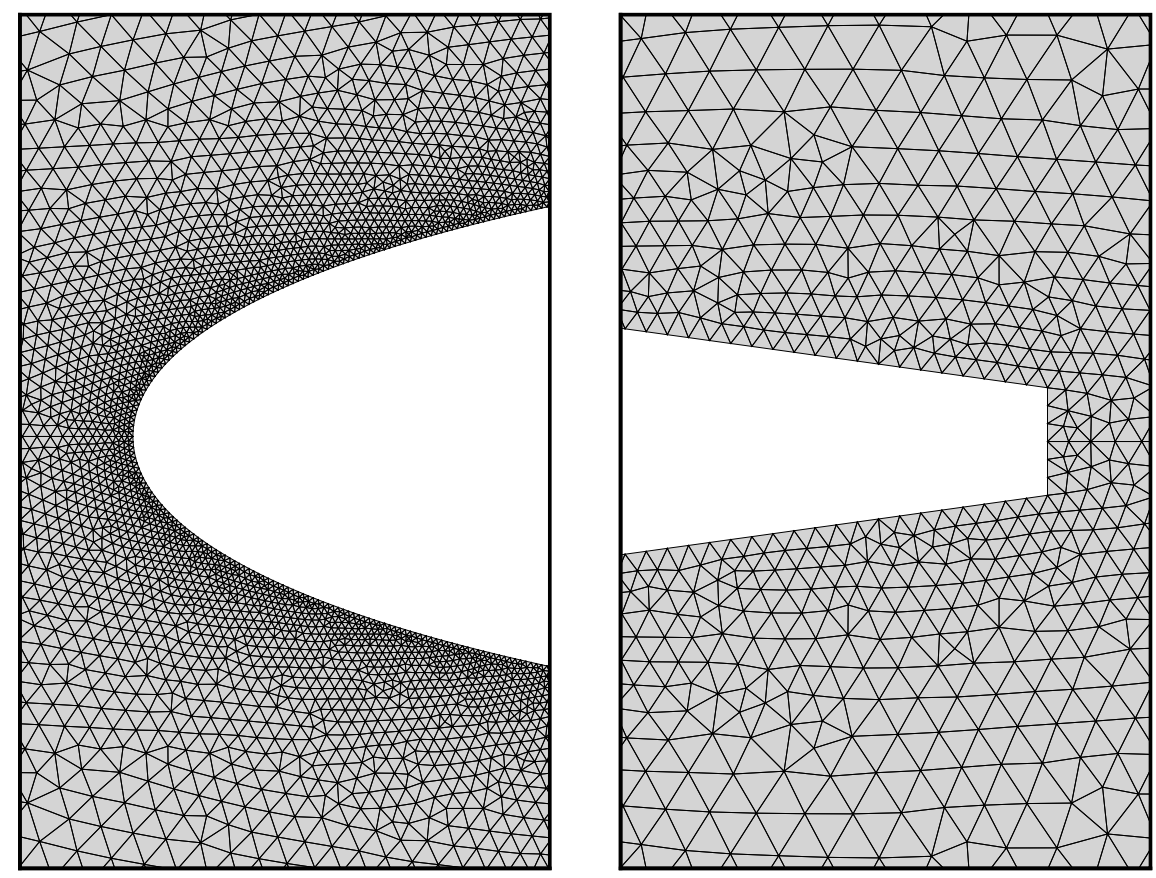

Figure 14: Body-fitted mesh for the NACA0012 airfoil with blunt trailing edge. Right: zoom on the leading edge. Left: zoom on the trailing edge.

The body-fitted and IB meshes are respectively shown in Figures 14 15. The meshes are refined at the leading and trailing edges and the far field is located at 50 chords from the airfoil. The same parameters are used to generate the IB and body-fitted meshes, ensuring comparable cell sizes and refinement. In terms of the chord $(c)$, the cell sizes at the wall, leading edge, trailing edge and far field are respectively: $5 \mathrm{e}-3 c, 1 \mathrm{e}-3 c, 5 \mathrm{e}-4 c$ and $4 c$. A linear cell size growth 
is allowed from the wall to the far field boundary. For the body-fitted and IB meshes, it gives a total of 36420 and 49136 cells respectively.
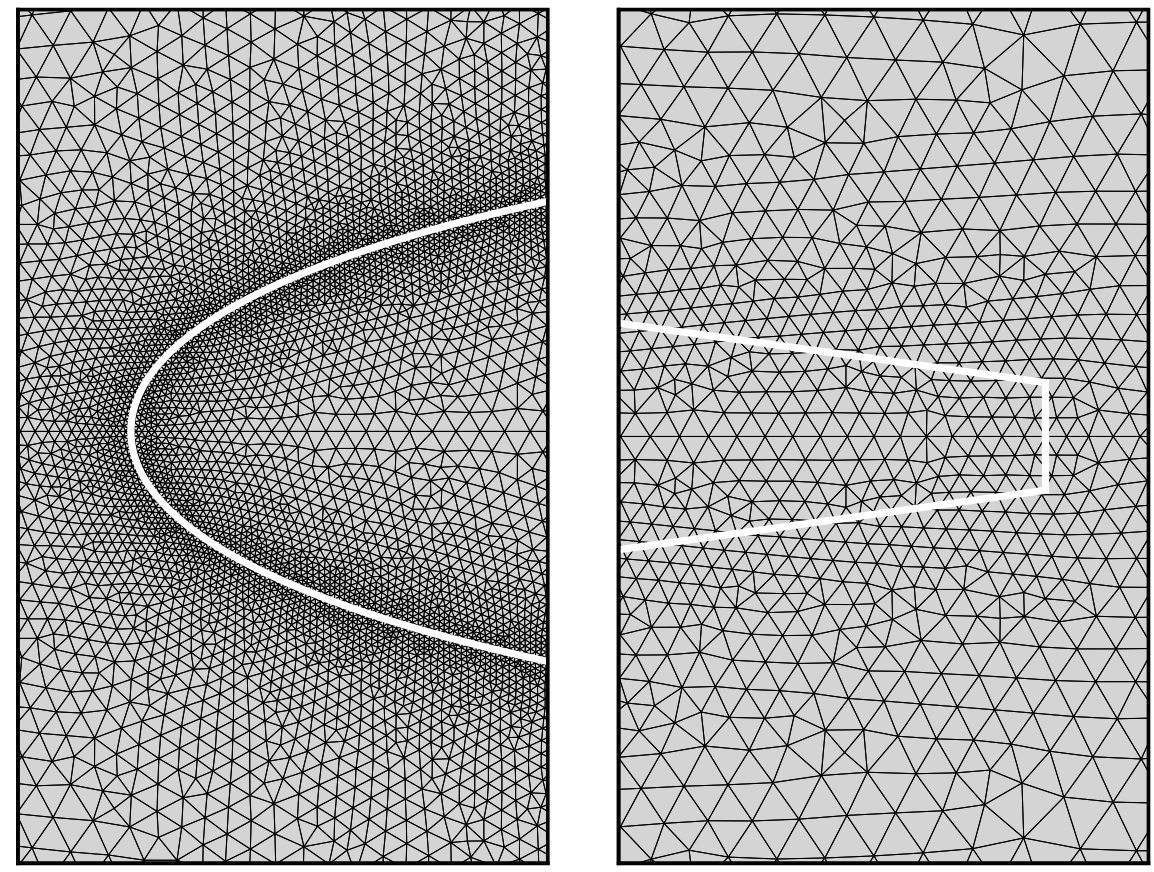

Figure 15: Immersed boundary mesh for the NACA0012 airfoil with blunt trailing edge. Right: zoom on the leading edge. Left: zoom on the trailing edge.

For this test case, the standard subsonic flow conditions of Mach $=0.5$ and $A o A=1.25 \mathrm{deg}$ are used [34]. To allow a fair comparison with the penalization methods, the body-fitted (BF) simulation is performed with both the ST and CCST wall boundary conditions. As a reminder, the CBVP and CBVP-Hs both account for the wall curvature, similar to the CCST. 


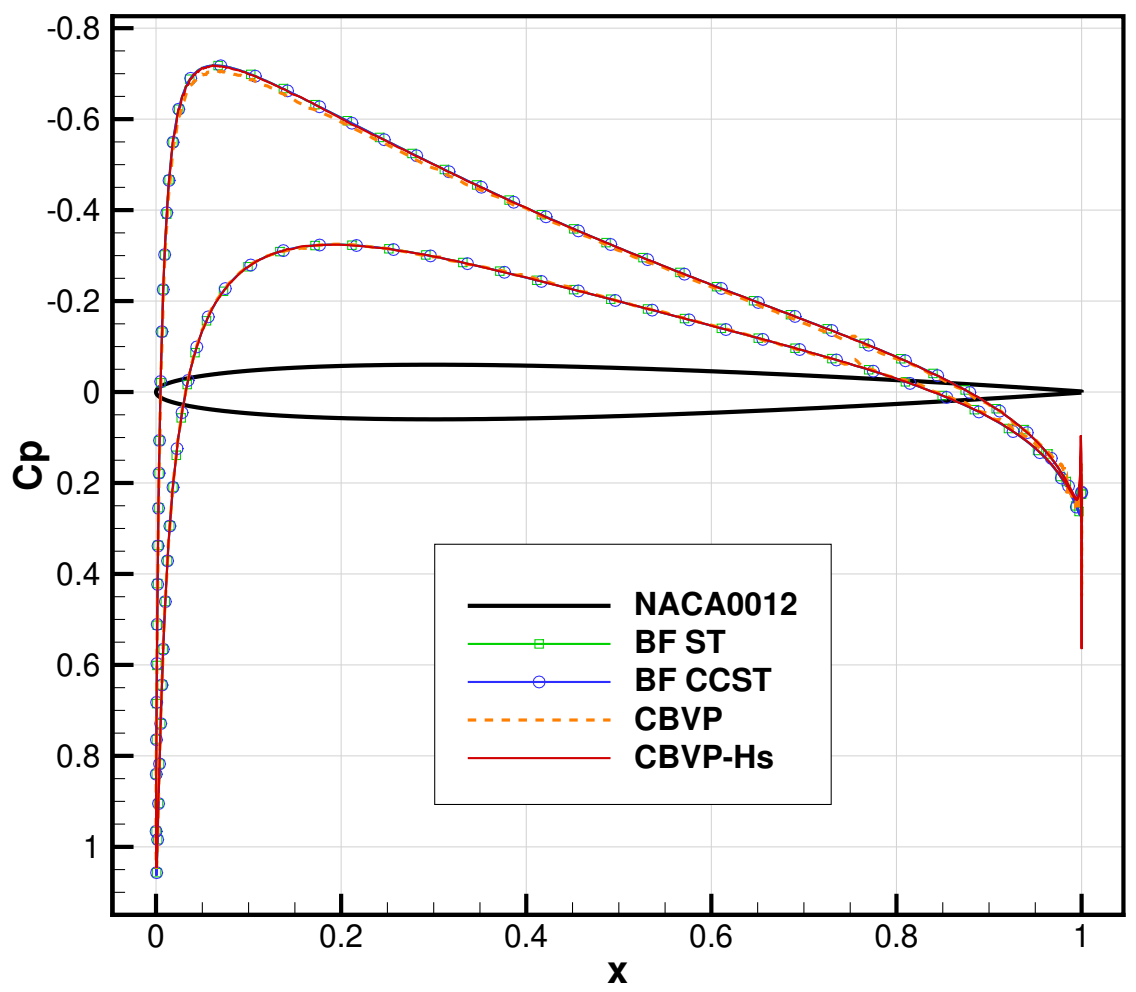

(a) Global view

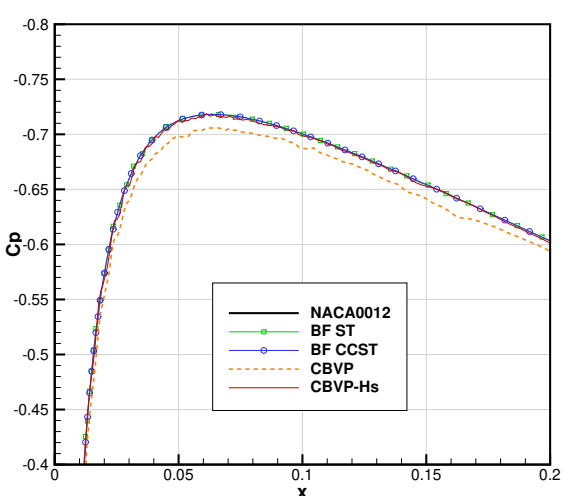

(b) Zoom near $C p_{\max }$

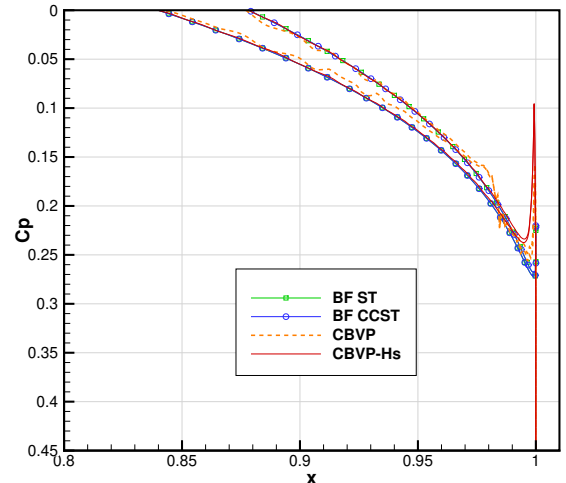

(c) Zoom at the trailing edge

Figure 16: Wall Cp for the NACA0012 airfoil at $\mathrm{Mach}=0.5$ and $\mathrm{AoA}=1.25 \mathrm{deg}$. Comparison to the BF ST and BF CCST. 
The results from Figure 16 illustrate the $C p$ distribution at the wall. For the body-fitted results, the ST and CCST provide a similar solution where the two $C p$ curves are superimposed on Figure $16 \mathrm{~b}$. On a geometry with moderate curvature $(\kappa)$ and using a mesh with adequate refinement $(\Delta x)$ both the ST and CCST are expected to be equivalent. This is justified by Eq. 20 which provides an error estimate for the pressure extrapolation $\Delta P / P_{w}$. When $\kappa \Delta x \rightarrow 0$, $\Delta P / P_{w} \rightarrow 0$ and the CCST and ST become equivalent. For this test case, the ST shows a maximum error of $\Delta P / P_{w} \approx 1 \%$ with $\gamma M_{w}^{2} \kappa=12.3$ and a wall cell size of $\Delta x=1 \mathrm{e}-3$. An error of $\approx 1 \%$ is deemed small enough to explain the superposition of the $C p$ curves for the ST and CCST in Figure 16. The lift coefficients for the body-fitted solutions are respectively $C_{L}=0.1776$ and $C_{L}=0.1777$ for the ST and CCST boundary conditions.

Now considering the penalization methods, the $C p$ distribution for the CBVP method is close to the body-fitted solution with a slight overestimation in the vicinity of the maximum suction. Also, the pressure coefficient is not as accurate at the trailing edge for this method. On the other hand, the $C p$ distribution for the CBVP-Hs fits well with the body-fitted results. The lift coefficients are respectively $C_{L}=0.1693$ and $C_{L}=0.1768$ for the CBVP and CBVP-Hs. Again the CBVP-Hs is much closer to the body-fitted solution $\left(C_{L} \in[0.1776,0.1777]\right)$. This result shows that this penalization method is able to perform well on an airfoil case if the mesh is sufficiently refined, especially around sharp features. For instance, it was observed that the trailing edge must contain at least 6 solid cells in its thickness for the penalization method to work well (Figure 15 ). In this way, the interpolation and gradient stencils use only valid solid cells and avoid the ones near the body centerline. The results also confirm that the penalization method is suitable for unstructured grids.

\subsection{Flow Around an Ice Horn}

Ice shapes can be very challenging for mesh generation and for the flow solver. One particular case that was found difficult to deal with in a previous communication [29] is the ice horn generated by the conditions listed in Table 
1. This ice shape exhibits an ice horn of high curvature which can lead to flow separation, even with a Euler flow solver. This behavior is shown in Figure 17. where the simulation is performed using the body-fitted mesh of Figure $18 \mathrm{a}$ and the ST wall boundary condition.

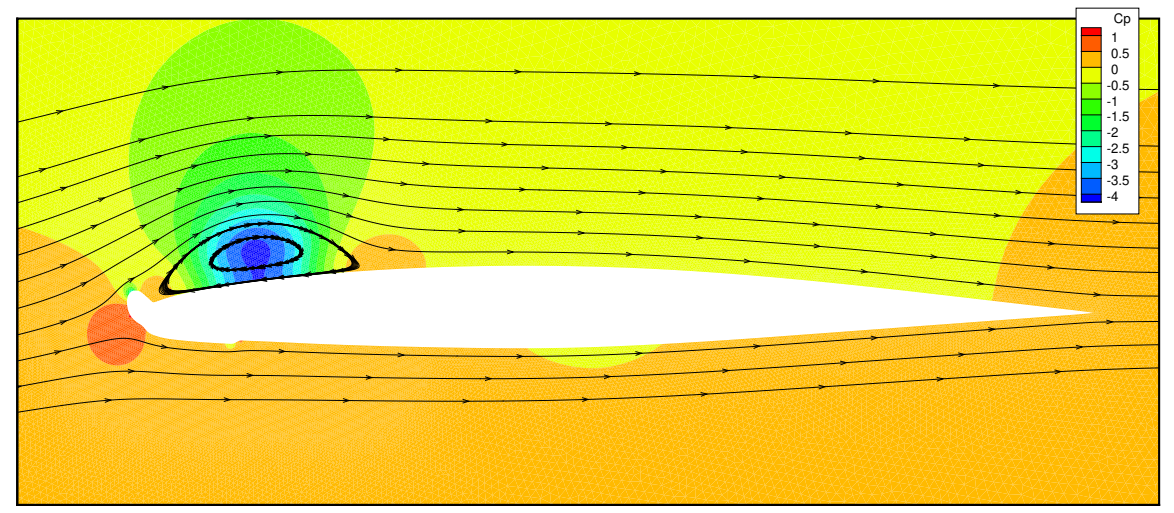

Figure 17: Field of Cp contours and velocity streamtraces for the ice horn case using a bodyfitted mesh and the ST wall boundary condition

For this test case, the strategy is to use a body fitted mesh for the clean geometry (GLC305) and to use an IB to account for the ice shape (Figure 18b). In this way, the sharp trailing edge is solved accurately and the ice shape can be dealt with to first order accuracy (penalization).

Here, the Euler flow is solved on the ice accreted GLC305 where the ice shape was generated with IGLOO2D for an icing time of $675 \mathrm{~s}$ (half the full ice accretion time). The body-fitted and IB meshes around the ice horn are shown in Figure 18. Again the same cell size is used in the vicinity of the wall for both cases to allow a fair comparison. For this test case, the wall $C p$ is converged to $10^{-6}$. 


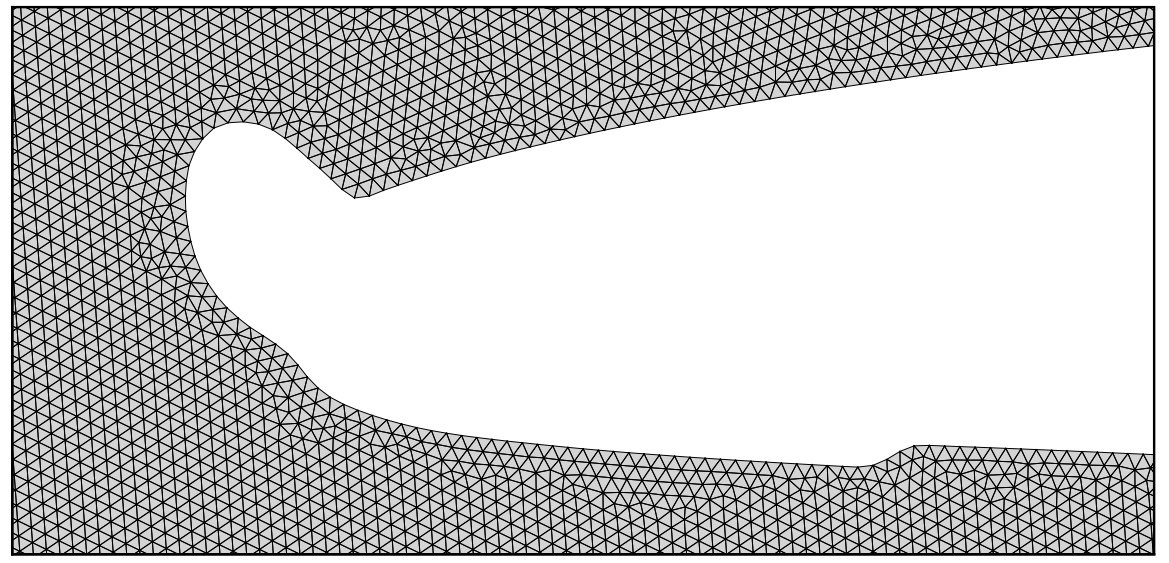

(a) Body-Fitted

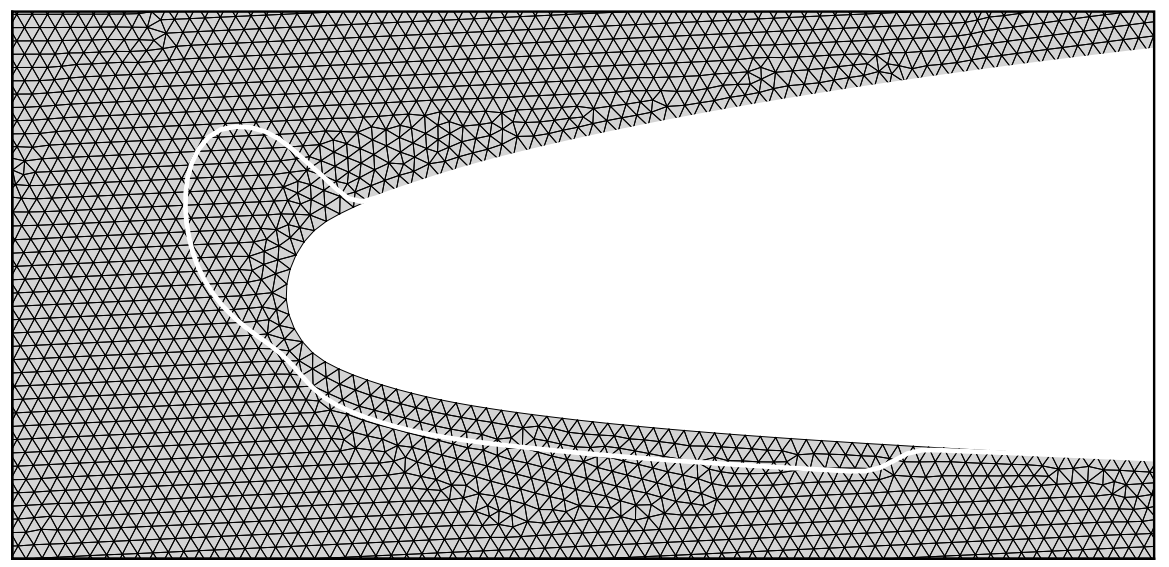

(b) Immersed Boundary

Figure 18: Mesh around the ice horn

The $C p$ distribution on the ice horn is compared in Figure 20. First, the body-fitted solutions using the two types of wall boundary conditions are examined: ST and CCST. The ST was previously shown to give a separated flow in Figure 17 and this can now be observed in Figure 20. This flow separation is induced by the numerical viscosity of the flow solver and has no physical meaning. It would be preferable to obtain an attached solution to be in line with the theory of inviscid flows. Numerical experiments showed that a finer mesh 
allows an attached solution to be obtained with the ST (Figure 21).

An alternative approach is to apply the CCST to account for the wall curvature and therefore increase the accuracy of the solution on coarser mesh. On Figures 1920 , it is shown that the use of the CCST indeed leads to an attached flow solution on the current mesh. This also significantly increases the suction peak at the ice horn tip which is now at $C p \approx-13$ in comparison to $C p \approx-3$ for the ST approach. As the mesh is refined (Figure 21), the ST solution tends toward the CCST solution except the suction peak is not fully recovered yet. Assuming an error of $1 \%$ is desired on the pressure extrapolation (Eq. 200), a cell size can be estimated for the ice horn. According to this approach, the cell size should be around 2e-5 for the ST and CCST to be equivalent.

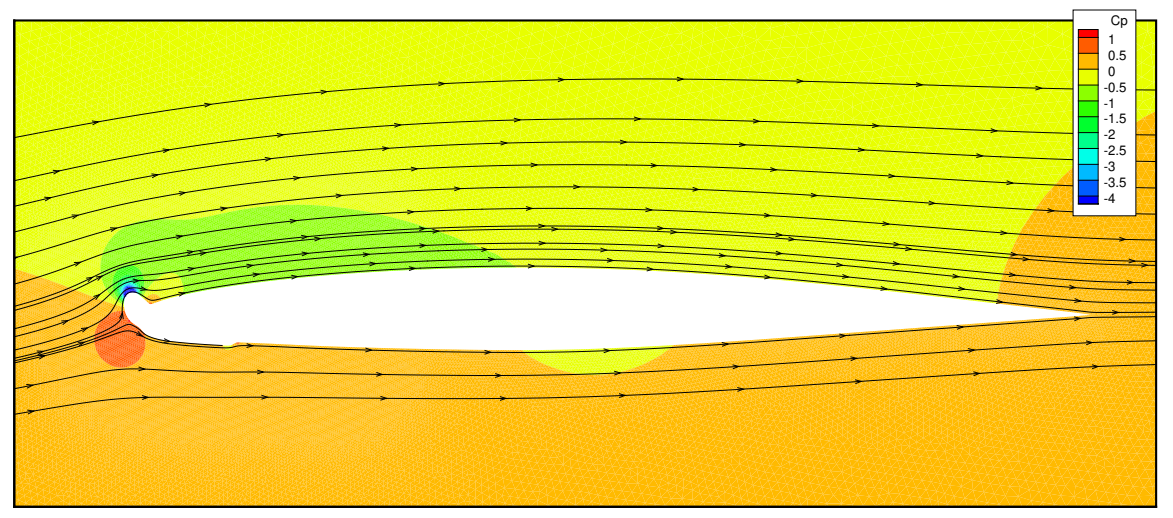

Figure 19: Field Cp contours and velocity streamtraces for the ice horn case using a body-fitted mesh and the CCST wall boundary condition 


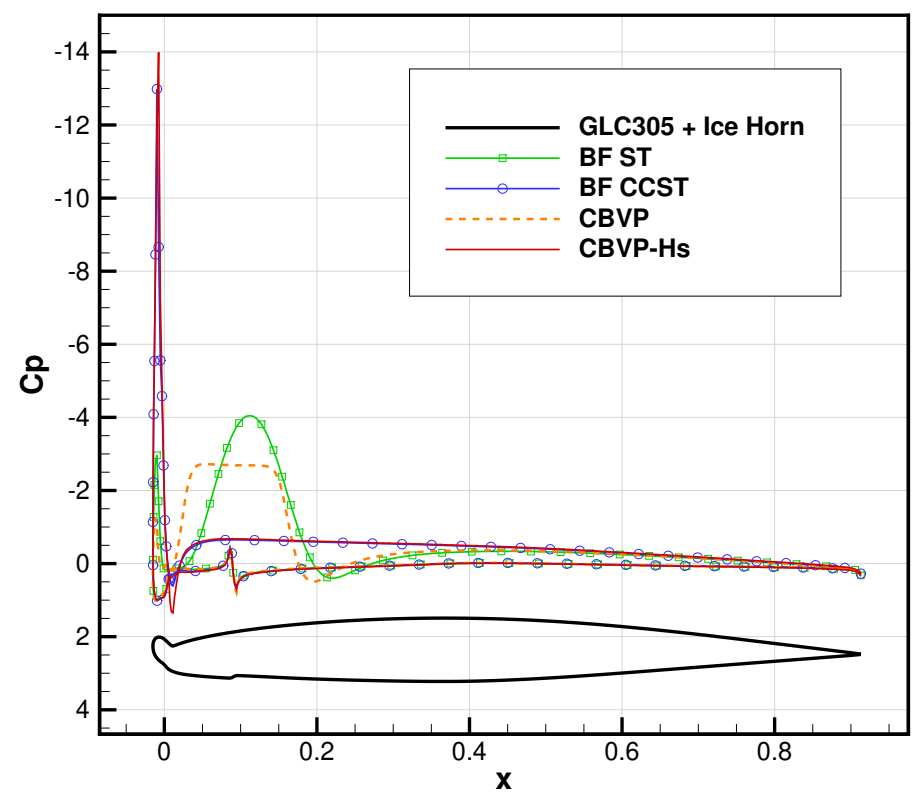

(a) Global view

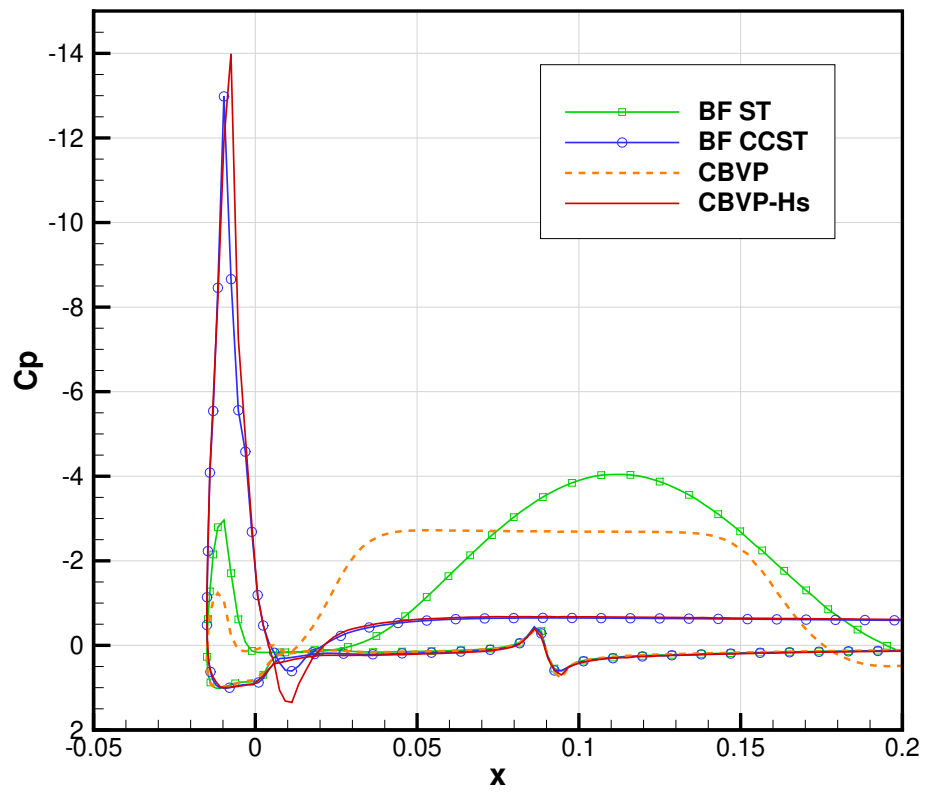

(b) Zoom near the ice horn

Figure 20: Wall $\mathrm{Cp}$ for the ice horn (horn tip cell size 2e-3). 


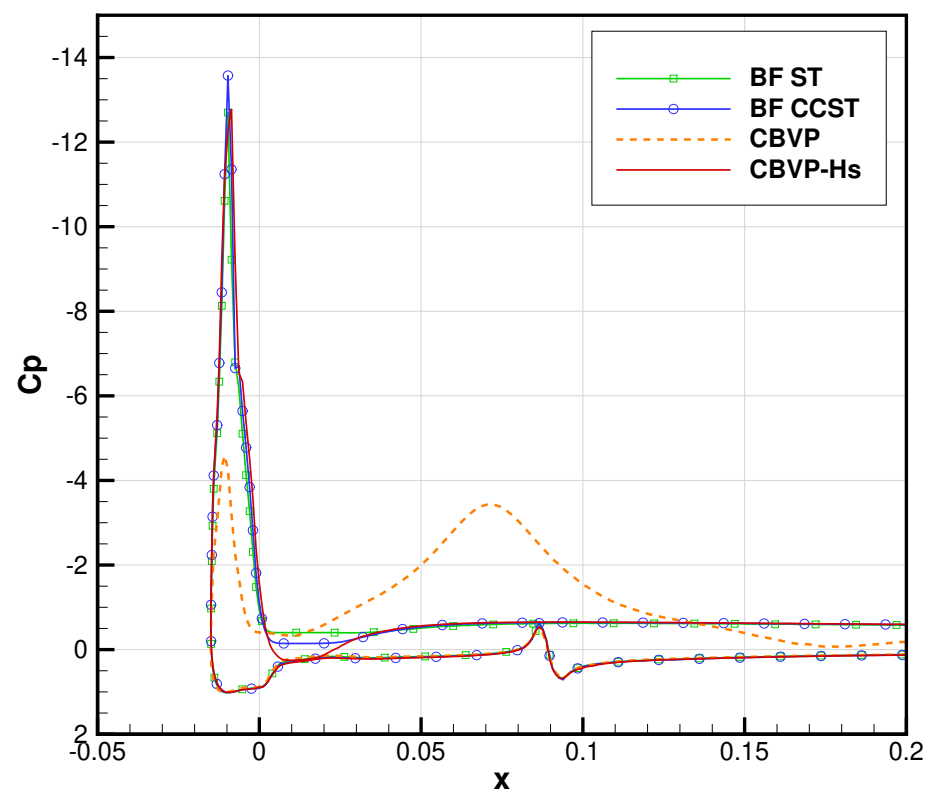

(a) horn tip cell size 1e-3

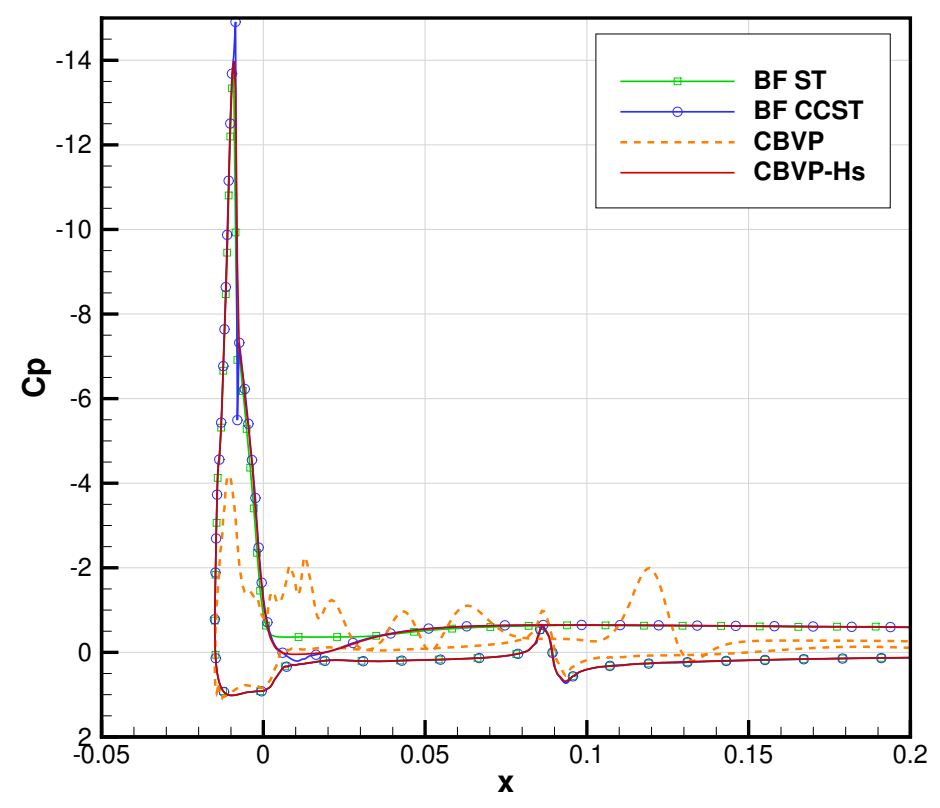

(b) horn tip cell size 5e-4

Figure 21: Wall $\mathrm{Cp}$ for the ice horn 
The results from the penalization methods are examined next: CBVP and CBVP-Hs. Both method account for the wall curvature in the imposition of the wall boundary condition. However, as shown on Figure 20, the CBVP still exhibits flow separation on the ice horn case while the CBVP-Hs does not. This behavior is similar to the results obtained earlier on the cylinder, where the CBVP-Hs was more accurate and behaved better aft of the cylinder for coarser meshes. Again, it suggests that the CBVP would give similar results if the mesh were refined. As the mesh is refined (Figure 21), the amplitude of the flow separation reduces at first (Figure 21a) and the suction peak increases. However by refining the mesh once more, the CBVP fails to converge to steady state, exhibiting unsteady flow behavior. This explains the poor CBVP solution on Figure 21b. In fact, it was difficult to converge the Cp further than 1e-4 for the CBVP method whereas the CBVP-Hs exhibited good convergence to steady state.

The difference between the two methods could be explained by the boundary conditions which conserve entropy and total enthalpy for the CBVP-Hs. Also the CBVP-Hs better impose the streamline conditions near the IB. The CBVPHs follows the boundary conditions imposed by the CCST and exhibits similar behavior on the ice horn case. From the previous observations, the CBVP-Hs is concluded to be more accurate and more suitable for geometries with high curvature, particularly on coarser meshes.

\subsection{Transonic Flow Around a NACA0012}

In order to show that the method is not only limited to subsonic flows, the transonic flow over a NACA0012 airfoil is computed. The flow parameters are selected as per 34, with $M a c h=0.8$ and $A o A=1.25 \mathrm{deg}$. The comparison is performed between the Body-Fitted mesh approach using the CCST and the CBVP-Hs on meshes of equivalent cell sizes. The mesh used are twice as fine as the ones used for the subsonic test case with the same refinements zones and geometry (i.e. NACA0012 with a blunt trailing edge).

The results of Figure 22 show that for a mesh of comparable refinement, 
the CBVP-Hs is able to capture the shock location with reasonable accuracy compared to the CCST results. The lift coefficient for the CBVP-Hs is $C_{L}=$ 0.3460 which is $1.6 \%$ off the body-fitted results $\left(C_{L}=0.3517\right)$. A better $C_{L}$ could be obtained by properly refining the mesh in the vicinity of the shocks. However, it still shows the potential of the CBVP-Hs for the simulation of transonic and supersonic flows.

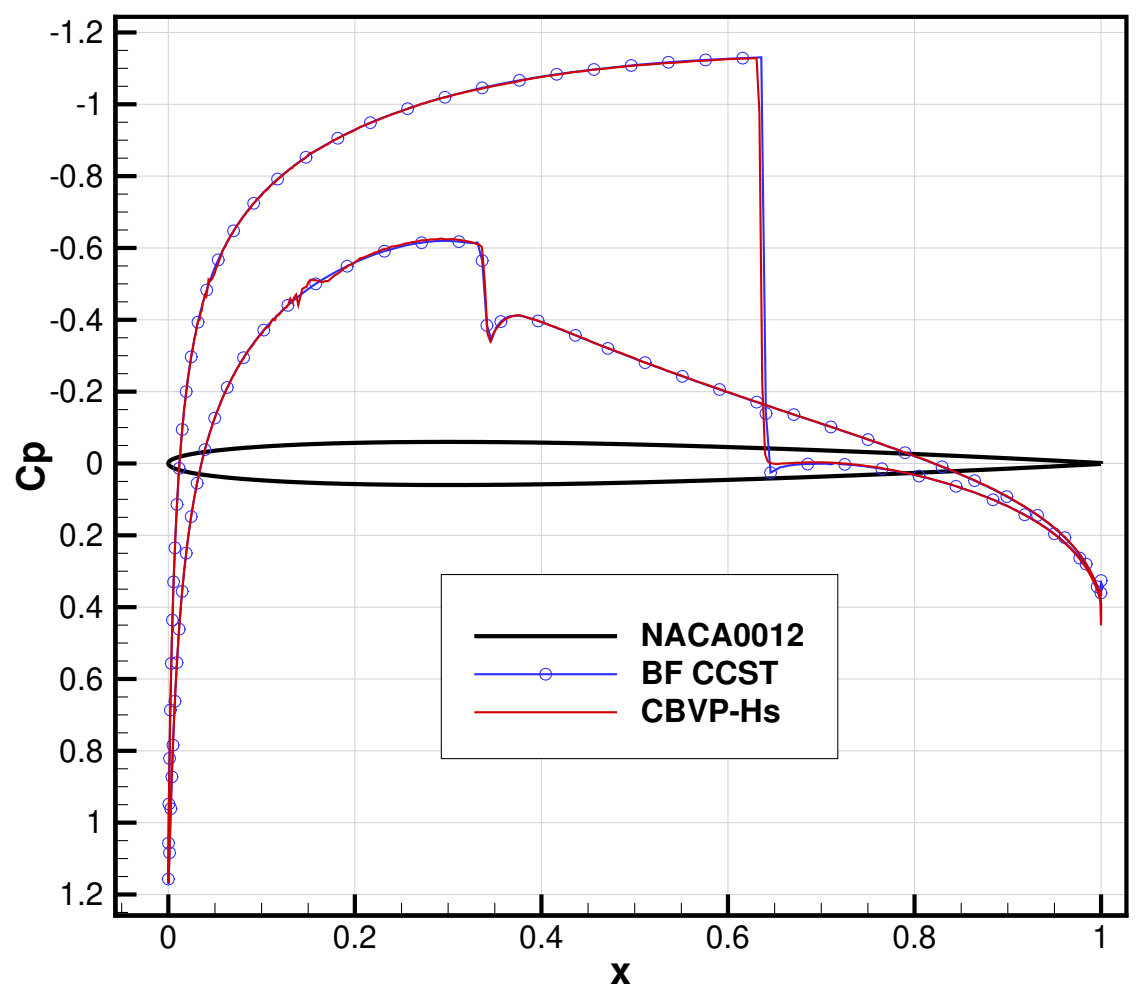

Figure 22: Wall $\mathrm{Cp}$ for the NACA0012 airfoil at $\mathrm{Mach}=0.8$ and $\mathrm{AoA}=1.25 \mathrm{deg}$. Comparison to the BF ST and BF CCST.

\section{Conclusion}

In this paper, a penalization method which conserves entropy and total enthalpy is developed for the Euler equations. 
Two types of numerical wall boundary conditions are described for bodyfitted meshes: the symmetry technique (ST) and curvature-corrected symmetry technique (CCST). For the immersed boundary method, the penalization is selected for its simplicity of implementation and its mesh and discretization independence. The penalization method is usually applied to the Navier-Stokes equations and applications to the Euler equations are few. The characteristicbased volume penalization (CBVP) of [13] showed to be suitable for most cases but exhibited flow separation in some icing application with high curvature geometries. A new method is thus developed, the CBVP-Hs, which can be seen as the application of the CCST boundary conditions using the penalization method.

The body-fitted and penalization methods are tested on four test cases. A mesh convergence is performed for the weakly compressible flow around a circular cylinder. Then the subsonic and transonic flows around a NACA0012 airfoil are solved. Also, the airflow around an ice horn of high curvature is evaluated to assess the methods on a challenging icing case.

The CBVP and CBVP-Hs are found to tend towards the same solution for the cylinder case. However, the CBVP method exhibits a higher error level on the pressure coefficient, conservation of total enthalpy and conservation of entropy compared to the CBVP-Hs. The CBVP-Hs is also found to be more accurate on coarser meshes. For instance, on the cylinder the CBVP-Hs solution on the $D / \Delta x=40$ mesh is equivalent to the CBVP on the $D / \Delta x=160$ mesh. For the ice horn case on a coarse mesh, the CBVP exhibits flow separation while the flow remains attached for the CBVP-Hs, which is more in line with inviscid flow theory. A mesh refinement was not sufficient to obtain an attached flow with the CBVP. Also, the CBVP-Hs shows a good potential for the simulation of transonic flows as it can capture the same shock location as a Body-Fitted simulation of comparable mesh refinement.

It is concluded that the application of the penalization method to the Euler equations requires special care to obtain accurate solutions on high curvature geometries. The newly developed approach (CBVP-Hs) proved to be a suitable 
candidate for this purpose. Although the method was presented in $2 \mathrm{D}$ in this article, no major difficulty is expected for the extension of this penalization method in 3D, which will be done in the future.

\section{Acknowledgments}

This work is supported by the Natural Sciences and Engineering Research Council of Canada (NSERC) and the ONERA, the French Aerospace Lab.

\section{References}

[1] R. Mittal, G. Iaccarino, Immersed boundary methods, Annual Review of Fluid Mechanics 37 (2005) 239-261. doi:10.1146/annurev.fluid.37. 061903.175743

[2] F. Sotiropoulos, X. Yang, Immersed boundary methods for simulating fluid-structure interaction, Progress in Aerospace Sciences 65 (2014) 1 21. doi:http://dx.doi.org/10.1016/j.paerosci.2013.09.003.

[3] P. Angot, C.-H. Bruneau, P. Fabrie, A penalization method to take into account obstacles in incompressible viscous flows, Numerische Mathematik 81 (4) (1999) 497-520. doi:10.1007/s002110050401

[4] J. P. Caltagirone, E. Arquis, Recirculations en milieu poreux, in: C. R. Acad. Sci. Paris, Vol. 302 of série II, 1986.

[5] K. Khadra, P. Angot, S. Parneix, J.-P. Caltagirone, Fictitious domain approach for numerical modelling of Navier-Stokes equations, International Journal for Numerical Methods in Fluids 34 (8) (2000) 651-684. doi: 10.1002/1097-0363(20001230)34:8<651: :AID-FLD61>3.0.CO;2-D.

URL http://dx.doi.org/10.1002/1097-0363(20001230)34:8<651:: AID-FLD61>3.0.C0;2-D 
[6] H. Beaugendre, F. Morency, F. Gallizio, S. Laurens, Computation of ice shedding trajectories using cartesian grids, penalization, and level sets, Model. Simul. Eng. 2011 (2011) 3:1-3:15. doi:10.1155/2011/274947.

[7] Q. Liu, O. V. Vasilyev, A Brinkman penalization method for compressible flows in complex geometries, Journal of Computational Physics 227 (2) (2007) 946 -966. doi:http://dx.doi.org/10.1016/j.jcp.2007.07.037

[8] A. Piquet, O. Roussel, A. Hadjadj, A comparative study of Brinkman penalization and direct-forcing immersed boundary methods for compressible viscous flows, Computers \& Fluids 136 (2016) 272 - 284. doi:https: //doi.org/10.1016/j.compfluid.2016.06.001

[9] O. Boiron, G. Chiavassa, R. Donat, A High-Resolution Penalization Method for large Mach number Flows in the presence of Obstacles, Computers and Fluids 38 (3) (2009) 703-714. doi:10.1016/j.compfluid.2008. 07.003

[10] R. Abgrall, H. Beaugendre, C. Dobrzynski, An immersed boundary method using unstructured anisotropic mesh adaptation combined with level-sets and penalization techniques, Journal of Computational Physics 257 (2014) 83 - 101. doi:http://dx.doi.org/10.1016/j.jcp.2013.08.052.

[11] L. Nouveau, Adaptive residual based schemes for solving the penalized Navier Stokes equations with moving bodies: application to ice shedding trajectories, Theses, Université de Bordeaux (Dec. 2016).

URL https://tel.archives-ouvertes.fr/tel-01500093

[12] I. Ramière, P. Angot, M. Belliard, A fictitious domain approach with spread interface for elliptic problems with general boundary conditions, Computer Methods in Applied Mechanics and Engineering 196 (4) (2007) 766 - 781. doi:https://doi.org/10.1016/j.cma.2006.05.012.

[13] E. Brown-Dymkoski, N. Kasimov, O. V. Vasilyev, A characteristic based volume penalization method for general evolution problems applied to com- 
pressible viscous flows, Journal of Computational Physics 262 (2014) 344 357. doi:https://doi.org/10.1016/j.jcp.2013.12.060

[14] Y. Bae, Y. J. Moon, On the use of Brinkman penalization method for computation of acoustic scattering from complex boundaries, Computers \& Fluids 55 (2012) 48 - 56 . doi:https: //doi.org/10.1016/j.compfluid.2011.10.015

URL http://www.sciencedirect.com/science/article/pii/ S0045793011003215

[15] E. Brown-Dymkoski, N. Kasimov, O. V. Vasilyev, A characteristic-based volume penalization method for arbitrary mach flows around solid obstacles, in: J. Fröhlich, H. Kuerten, B. J. Geurts, V. Armenio (Eds.), Direct and Large-Eddy Simulation IX, Springer International Publishing, Cham, 2015, pp. 109-115.

[16] A. Dadone, B. Grossman, Surface boundary conditions for the numerical solution of the euler equations, AIAA Journal 32 (2) (1994) 285-293. doi: $10.2514 / 3.11983$

[17] W. B. Wright, User manual for the nasa glenn ice accretion code lewice version 2.2.2, Tech. rep., NASA, nASA/CR-2002-211793 (2002).

URL https://ntrs.nasa.gov/archive/nasa/casi.ntrs.nasa.gov/ 20020080990.pdf

[18] P. Trontin, G. Blanchard, A. Kontogiannis, P. Villedieu, Description and assessment of the new onera 2D icing suite IGLOO2D, in: 9th AIAA Atmospheric and Space Environments Conference, 2017. doi:10.2514/6. 2017-3417.

[19] F. Capizzano, A compressible flow simulation system based on cartesian grids with anisotropic refinements, in: Aerospace Sciences Meetings, American Institute of Aeronautics and Astronautics, 2007, pp.--. doi:10.2514/6.2007-1450 
[20] A. Dadone, B. Grossman, Ghost-cell method for analysis of inviscid threedimensional flows on cartesian-grids, Computers \& Fluids 36 (10) (2007) 1513 - 1528, special Issue Dedicated to Professor Michele Napolitano on the Occasion of his 60th Birthday. doi:https://doi.org/10.1016/j. compfluid.2007.03.013.

[21] A. Dadone, B. Grossman, Ghost-cell method for inviscid two-dimensional flows on cartesian grids, AIAA Journal 42 (12) (2004) 2499-2507. doi: http://dx.doi.org/10.2514/1.697

[22] Z. J. Wang, Y. Sun, Curvature-based wall boundary condition for the euler equations on unstructured grids, AIAA Journal 41 (1) (2003) 27-33. doi: $10.2514 / 2.1931$.

[23] P. J. Schneider, D. H. Eberly, Chapter 13 - computational geometry topics, in: D. H. SCHNEIDER, PHILIP J.and EBERLY (Ed.), Geometric Tools for Computer Graphics, The Morgan Kaufmann Series in Computer Graphics, Morgan Kaufmann, San Francisco, 2003, pp. 673 - 825. doi:https://doi. org/10.1016/B978-155860594-7/50016-3

[24] A. Sarthou, S. Vincent, J. P. Caltagirone, P. Angot, Eulerian-lagrangian grid coupling and penalty methods for the simulation of multiphase flows interacting with complex objects, International Journal for Numerical Methods in Fluids 56 (8) (2008) 1093-1099. doi:10.1002/fld.1661.

[25] P. Roe, Approximate riemann solvers, parameter vectors, and difference schemes, Journal of Computational Physics 43 (2) (1981) 357 - 372. doi: https://doi.org/10.1016/0021-9991(81)90128-5.

[26] T. Barth, D. Jespersen, The design and application of upwind schemes on unstructured meshes, in: 27th Aerospace Sciences Meeting, 1989. doi: $10.2514 / 6.1989-366$

[27] A. Etcheverlepo, Développement de méthodes de domaines fictifs au second ordre, Ph.D. thesis, Université Bordeaux I (2013). 
URL http://ori-oai.u-bordeaux1.fr/pdf/2013/ETCHEVERLEPO_ ADRIEN_2013.pdf

[28] A. Sarthou, S. Vincent, J. Caltagirone, A second-order curvilinear to cartesian transformation of immersed interfaces and boundaries. application to fictitious domains and multiphase flows, Computers \& Fluids 46 (1) (2011) 422 - 428, 10th ICFD Conference Series on Numerical Methods for Fluid Dynamics (ICFD 2010). doi:http://dx.doi.org/10.1016/j. compfluid.2010.11.008.

[29] P. Lavoie, G. Blanchard, E. Radenac, E. Laurendeau, P. Villedieu, A penalization method for $2 \mathrm{~d}$ ice accretion simulations, in: SAE Technical Paper, SAE International, 2019. doi:10.4271/2019-01-1939

[30] R. Mittal, H. Dong, M. Bozkurttas, F. Najjar, A. Vargas, A. von Loebbecke, A versatile sharp interface immersed boundary method for incompressible flows with complex boundaries, Journal of Computational Physics 227 (10) (2008) 4825 - 4852. doi:http://dx.doi.org/10.1016/j.jcp.2008.01. 028.

[31] D. K. Clarke, H. A. Hassan, M. D. Salas, Euler calculations for multielement airfoils using cartesian grids, AIAA Journal 24 (3) (1986) 353-358. doi: $10.2514 / 3.9273$.

[32] M. Berger, M. Aftosmis, Progress towards a cartesian cut-cell method for viscous compressible flow, in: 50th AIAA Aerospace Sciences Meeting, 2012, aIAA 2012-1301. doi:doi.org/10.2514/6.2012-1301.

[33] C. L. Ladson, C. W. B. Jr., A. S. Hill, D. W. Sproles, Computer program to obtain ordinates for naca airfoils, Tech. rep., NASA, nASA-TM-4741 (1996).

URL https://ntrs.nasa.gov/citations/19970008124

[34] J. C. Vassberg, A. Jameson, In pursuit of grid convergence for two- 
dimensional euler solutions, Journal of Aircraft 47 (4) (2010) 1152-1166.

doi: $10.2514 / 1.46737$. 